DOI 10.4171/JEMS/194

Benjamin D. Miller · Christian Rosendal

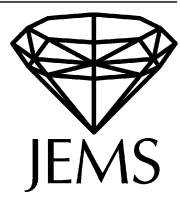

\title{
Descriptive Kakutani equivalence
}

Received October 10, 2006 and in revised form September 28, 2008

\begin{abstract}
We consider a descriptive set-theoretic analog of Kakutani equivalence for Borel automorphisms of Polish spaces. Answering a question of Nadkarni, we show that up to this notion, there are exactly two aperiodic Borel automorphisms of uncountable Polish spaces. Using this, we classify all Borel $\mathbb{R}$-flows up to $C^{\infty}$-time-change isomorphism. We then extend the notion of descriptive Kakutani equivalence to all (not necessarily injective) Borel functions, and provide a variety of results leading towards a complete classification. The main technical tools are a series of Glimm-Effros and Dougherty-Jackson-Kechris-style embedding theorems.
\end{abstract}

Keywords. Borel functions, Borel $\mathbb{R}$-flows, Kakutani equivalence

\section{Contents}

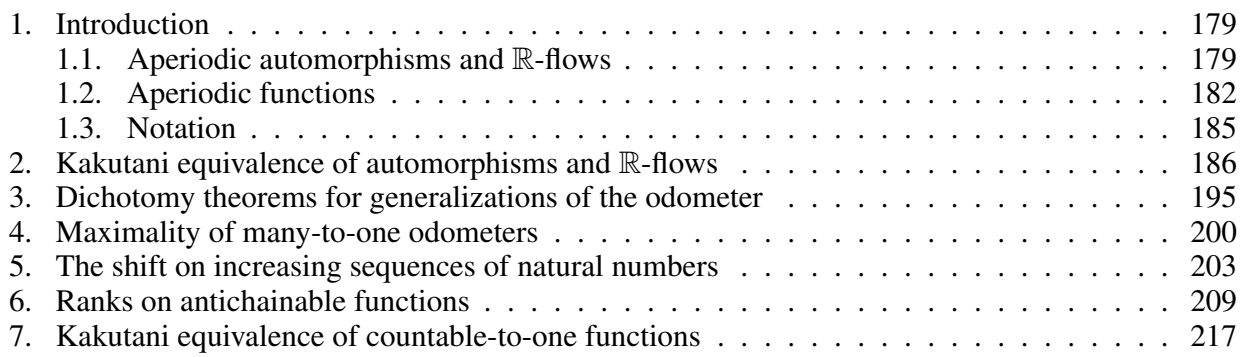

\section{Introduction}

\subsection{Aperiodic automorphisms and $\mathbb{R}$-flows}

Suppose that $X$ is a Polish space and $f: X \rightarrow X$ is Borel. The forward $(f$-)orbit of $x$ is given by $[x]_{f}=\left\{f^{n}(x): n \in \mathbb{N}\right\}$, and the $\left(f\right.$-)orbit of $x$ is given by $[x]_{f}=$

B. D. Miller: 8159 Constitution Road, Las Cruces, NM 88007, USA;

e-mail: glimmeffros@gmail.com

C. Rosendal: Department of Mathematics, Statistics, and Computer Science (M/C 249),

University of Illinois at Chicago, 851 S. Morgan Street, Chicago, IL 60607-7045, USA;

e-mail: rosendal@math.uic.edu

Mathematics Subject Classification (2010): Primary 03E15 
$\bigcup_{n \in \mathbb{N}} f^{-n}\left([x]_{f}\right)$. We say that $f$ is periodic if all of its forward orbits are finite, and we say that $f$ is aperiodic if all of its forward orbits are infinite.

A set $A \subseteq X$ is $(f$-)complete if it contains (perhaps infinitely many) points of every orbit. A set $A \subseteq X$ is $\left(f\right.$-)recurrent if for all $x \in A$, there exists $n \in \mathbb{Z}^{+}$such that $f^{n}(x) \in A$. When $f$ is aperiodic, this means exactly that $A$ intersects the forward orbit of every point of $A$ in an infinite set. The induced transformation associated with a recurrent set $A \subseteq X$ is the map $f_{A}: A \rightarrow A$ given by $f_{A}(x)=f^{n}(x)$, where $n \in \mathbb{Z}^{+}$is least such that $f^{n}(x) \in A$. It is not difficult to see that if $A \subseteq X$ is a recurrent Borel set, then $f_{A}$ is Borel.

A set $A \subseteq X$ is a(n) $(f$-)transversal if it contains exactly one point of every orbit. We say that $f$ is smooth if it has a Borel transversal. We regard smooth functions as being descriptive set-theoretically trivial.

We say that Borel functions $f: X \rightarrow X$ and $g: Y \rightarrow Y$ are Borel isomorphic, or $f \cong_{B} g$, if there is a Borel isomorphism $\pi: X \rightarrow Y$ such that $\pi \circ f=g \circ \pi$. All aperiodic, smooth Borel automorphisms of uncountable Polish spaces are Borel isomorphic. On the other hand, Clemens [2] has shown that the Borel isomorphism equivalence relation on non-smooth Borel automorphisms is very complex. Here we consider a natural weakening of Borel isomorphism.

We say that Borel functions $f$ and $g$ are (descriptively) Kakutani equivalent, or $f \approx_{K}$ $g$, if there are complete, recurrent Borel sets $A \subseteq X$ and $B \subseteq Y$ such that $f_{A} \cong_{B} g_{B}$. This notion is primarily of interest for aperiodic Borel functions, as the fact that all periodic Borel functions are smooth easily implies that the Kakutani equivalence class of a periodic Borel function depends only upon the cardinality of the corresponding set of orbits. Nadkarni [15] introduced the notion of Kakutani equivalence for Borel automorphisms. Actually, he required that both $A$ and $B$ are birecurrent (i.e., recurrent with respect to both the function and its inverse), but, since only smooth functions have complete Borel sets which are not birecurrent on any orbit, it is easy to see that his notion is equivalent to ours. While an elementary argument going back to von Neumann (see $\$ 7.18$ of [15]) shows that Kakutani equivalence of Borel automorphisms is indeed an equivalence relation, we do not know if this generalizes even to two-to-one Borel functions.

Descriptive Kakutani equivalence originates in an ergodic-theoretic notion due to Kakutani [10], which he conjectured to be trivial. While this conjecture turned out to be incorrect, the following theorem confirms its descriptive set-theoretic analog, and gives also an affirmative answer to Nadkarni's question [15] as to whether all aperiodic, nonsmooth, rank one Borel automorphisms are Kakutani equivalent:

Theorem A. All aperiodic, non-smooth Borel automorphisms of Polish spaces are Kakutani equivalent.

There is also a "continuous" version of Theorem A but before describing this, it will be useful to view first Kakutani equivalence in a somewhat different light. Associated with every orbit $[x]_{f}$ is the quasi-order $\leq_{f}$ on $[x]_{f}$ given by

$$
x \leq_{f} y \Leftrightarrow \exists n \in \mathbb{N}\left(f^{n}(x)=y\right) .
$$


It is straightforward to check that $f: X \rightarrow X$ is Kakutani equivalent to $g: Y \rightarrow Y$ if and only if there are complete, recurrent Borel sets $A \subseteq X$ and $B \subseteq Y$ such that $\leq_{f} \mid A$ and $\leq_{g} \mid B$ are Borel isomorphic, in the sense that there is a Borel isomorphism $\pi: A \rightarrow B$ such that $x_{1} \leq_{f} x_{2} \Leftrightarrow \pi\left(x_{1}\right) \leq_{g} \pi\left(x_{2}\right)$, for all $x_{1}, x_{2} \in A$.

Suppose now that $\mathbb{R}$ acts in a Borel fashion on $X$, in which case we say also that $X$ is a Borel $\mathbb{R}$-flow. The (X-)orbit of $x$ is given by $[x]_{\mathbb{R}}=\{r+x: r \in \mathbb{R}\}$. Associated with every orbit $[x]_{\mathbb{R}}$ is the quasi-order $\leq_{X}$ on $[x]_{\mathbb{R}}$ given by

$$
x_{1} \leq_{X} x_{2} \Leftrightarrow \exists r \geq 0\left(r+x_{1}=x_{2}\right) .
$$

We say that a set $A \subseteq X$ is a(n) (X-)transversal if it contains exactly one point of every orbit, and we say that $X$ is smooth if it has a Borel transversal. The standard example of a non-smooth Borel $\mathbb{R}$-flow is irrational rotation on the torus. While the Borel isomorphism class of a free, smooth Borel $\mathbb{R}$-flow depends only upon the cardinality of the corresponding set of orbits, the Borel isomorphism equivalence relation on free, non-smooth Borel $\mathbb{R}$-flows is again quite complex.

A question dating back to Poincaré is that of determining whether two given compact, continuous $\mathbb{R}$-flows are topologically time-change isomorphic, in the sense that there is a homeomorphism of the underlying spaces $X$ and $Y$ which sends $\leq_{X}$ to $\leq_{Y}$. The analogous question can be posed in the purely descriptive set-theoretic setting, where we say that two free Borel $\mathbb{R}$-flows $X$ and $Y$ are (descriptively) time-change isomorphic if there is a Borel isomorphism $\pi: X \rightarrow Y$ such that $x_{1} \leq_{X} x_{2} \Leftrightarrow \pi\left(x_{1}\right) \leq_{Y} \pi\left(x_{2}\right)$, for all $x_{1}, x_{2} \in X$. By combining Theorem A with Wagh's theorem [21], we obtain:

Theorem B. All free, non-smooth Borel $\mathbb{R}$-flows on Polish spaces are time-change isomorphic.

In fact, it is not difficult to see that the time-change isomorphism can be chosen in such a way that for each $x \in X$, the map $f_{x}: \mathbb{R} \rightarrow \mathbb{R}$ defined implicitly by $\pi(r+x)=$ $f_{x}(r)+\pi(x)$ is $C^{\infty}$. There is also a straightforward extension of the above definition of time-change isomorphism to all (not necessarily free) Borel $\mathbb{R}$-flows, and the above theorem then gives rise to a classification of all Borel $\mathbb{R}$-flows.

The notion of $C^{\infty}$-time-change isomorphism makes sense also for free actions of $\mathbb{R}^{d}$. Surprisingly, in the measure-theoretic setting, the case $d \geq 2$ gives rise to a simpler equivalence relation than the case $d=1$. In fact, work of Feldman [5, 6] and Rudolph [17] implies that there is only one equivalence class when $d \geq 2$, while work of OrnsteinRudolph-Weiss [16] ensures that there is a continuum of possibilities when $d=1$. Although the equivalence relation trivializes in the descriptive set-theoretic setting when $d=1$, the higher dimensional analog remains open:

Problem C. Classify free Borel $\mathbb{R}^{d}$-actions on Polish spaces up to $\left(C^{\infty}\right.$-)time-change isomorphism.

In $\S 2$, we prove Theorems $\mathrm{A}$ and $\mathrm{B}$ (in fact, we prove a recent topological strengthening of Theorem Adue to Boykin-Jackson [1]). Although these are perhaps the most quotable results of the paper, we actually obtained them some time ago, and have since embarked upon the project of classifying all Borel functions up to Kakutani equivalence. In the remainder of the paper, we discuss various results in this direction. 


\subsection{Aperiodic functions}

We say that $f: X \rightarrow X$ is Kakutani embeddable into $g: Y \rightarrow Y$, or $f \sqsubseteq K g$, if there is a recurrent Borel set $B \subseteq Y$ such that $f \cong_{B} g_{B}$. We say that $f$ and $g$ are Kakutani bi-embeddable, or $f \cong_{K} g$, if $f \sqsubseteq_{K} g$ and $g \sqsubseteq_{K} f$.

We say that a set $A \subseteq X$ is $(f$-)stable if $f(A) \subseteq A$. We say that $f: X \rightarrow X$ is Kakutani reducible to $g: Y \rightarrow Y$, or $f \leq_{K} g$, if there is a complete, stable Borel set $A \subseteq X$ such that $f_{A} \sqsubseteq_{K} g$. We say that $f$ and $g$ are Kakutani bi-reducible, or $f \simeq_{K} g$, if $f \leq_{K} g$ and $g \leq_{K} f$.

It is clear that if $f \cong_{K} g$, then $f \simeq_{K} g$, and a simple Schröder-Bernstein argument shows that if $f \simeq_{K} g$, then $f \approx_{K} g$ (see Lemma 2.13). While the converses hold for Borel automorphisms, they are not true in general.

Given a property $P$ of Borel functions, we say that $f$ is essentially $P$ if there is a complete, recurrent Borel set $B$ such that $f_{B}$ has property $P$. In $\S 2$, we also show:

Theorem D. All aperiodic, essentially injective, non-smooth Borel functions on Polish spaces are Kakutani bi-reducible. Moreover, the class of such functions is closed under Kakutani equivalence.

The odometer is the isometry of $2^{\mathbb{N}}$ given by

$$
\sigma(x)= \begin{cases}0^{n} 1 y & \text { if } x=1^{n} 0 y \\ 0^{\infty} & \text { if } x=1^{\infty}\end{cases}
$$

A straightforward Baire category argument shows that the odometer is non-smooth (see Proposition 3.4). In fact, Shelah-Weiss [18] have shown that a Borel automorphism is non-smooth if and only if the odometer is Kakutani embeddable into it.

We say that a set $A \subseteq X$ is an ( $f$-)antichain if $x_{1} \leq_{f} x_{2} \Rightarrow x_{1}=x_{2}$, for all $x_{1}, x_{2} \in A$. We say that $f$ is antichainable if $X$ is the union of countably many Borel antichains. Note that a Borel injection is antichainable if and only if it is smooth. In $\S 3$, we establish the following generalization of the Shelah-Weiss theorem:

Theorem E. Suppose that $f$ is a Borel function on a Polish space. Then exactly one of the following holds:

(1) The function $f$ is antichainable.

(2) There is a continuous Kakutani embedding of $\sigma$ into $f$.

Given $f: X \rightarrow X$ and $g: Y \rightarrow Y$, let $f \oplus g$ denote the function on the disjoint union of $X$ and $Y$ which agrees with $f$ on $X$ and with $g$ on $Y$. Given properties $P$ and $Q$ of Borel functions, we say that a function can be decomposed into $P$ and $Q$ parts if it is Borel isomorphic to a function of the form $f \oplus g$, where $f$ has property $P$ and $g$ has property $Q$. In $\S 3$, we also introduce a two-to-one analog $\sigma_{2}$ of the odometer, and we establish another Shelah-Weiss-style dichotomy theorem:

Theorem F. Suppose that $f$ is a Borel function on a Polish space. Then exactly one of the following holds: 
(1) The function $f$ can be decomposed into antichainable and essentially injective parts.

(2) There is a continuous Kakutani embedding of $\sigma_{2}$ into $f$.

For each limit ordinal $\alpha<\omega_{1}$, the unilateral shift on $\alpha^{\mathbb{N}}$ is given by $s_{\alpha}\left(\left\langle x_{n}\right\rangle_{n \in \mathbb{N}}\right)=$ $\left\langle x_{n+1}\right\rangle_{n \in \mathbb{N}}$. We also use $s_{\mathbb{N}}$ to denote $s_{\omega}$. Let $s_{(\mathbb{N})}$ denote the restriction of $s_{\mathbb{N}}$ to the set $(\mathbb{N})^{\mathbb{N}}$ of injective sequences of natural numbers. As the antichains $A_{n}=\left\{x \in(\mathbb{N})^{\mathbb{N}}\right.$ : $x(0)=n$ cover $(\mathbb{N})^{\mathbb{N}}$, it follows that $s_{(\mathbb{N})}$ is antichainable.

We say that $f: X \rightarrow X$ is Borel embeddable into $g: Y \rightarrow Y$, or $f \sqsubseteq B g$, if there is a Borel injection $\pi: X \rightarrow Y$ such that $\pi \circ f=g \circ \pi$. In $\S 3$, we also establish a maximality result for the injective shift:

Theorem G. Every antichainable, aperiodic, countable-to-one Borel function on a Polish space is Borel embeddable into $s_{(\mathbb{N})}$.

In $\S 4$, we prove a maximality result for the two-to-one analog of the odometer:

Theorem H. Every aperiodic, countable-to-one Borel function on a Polish space is Kakutani embeddable into $\sigma_{2}$.

By combining Theorems $\mathrm{F}$ and $\mathrm{H}$, we obtain the following:

Theorem I. All aperiodic, essentially countable-to-one Borel functions on Polish spaces which cannot be decomposed into antichainable and essentially injective parts are Kakutani bi-reducible. Moreover, the class of such functions is closed under Kakutani equivalence.

We say that a function $f$ is well-founded if there is no sequence $\left\langle x_{n}\right\rangle_{n \in \mathbb{N}}$ such that $x_{n}=f\left(x_{n+1}\right)$ for all $n \in \mathbb{N}$. It is easy to see that every well-founded Borel function is antichainable.

For each limit ordinal $\alpha<\omega_{1}$, let $s_{[\alpha]}$ denote the restriction of $s_{\alpha}$ to the set $[\alpha]^{\mathbb{N}}$ of strictly increasing sequences of ordinals strictly less than $\alpha$. It is clear that these functions are well-founded. We also use $s_{[\mathbb{N}]}$ and $[\mathbb{N}]^{\mathbb{N}}$ to denote $s_{[\omega]}$ and $[\omega]^{\mathbb{N}}$. In $\S 5$, we show that $s_{[\mathbb{N}]}$ is the minimal obstruction to essential injectivity:

Theorem J. Suppose that $f$ is a Borel function on a Polish space. Then exactly one of the following holds:

(1) The function $f$ is essentially injective.

(2) There is a continuous Kakutani embedding of $s_{[\mathbb{N}]}$ into $f$.

By combining Theorems E and J we obtain a characterization of smoothness:

Theorem K. Suppose that $f$ is a Borel function on a Polish space. Then exactly one of the following holds:

(1) The function $f$ is smooth.

(2) There is a continuous Kakutani embedding of $\sigma$ or $s_{[\mathbb{N}]}$ into $f$.

We also obtain a maximality result for the increasing shift: 
Theorem L. Suppose that $f$ is an aperiodic Borel function on a Polish space. Then the following are equivalent:

(1) The function $f$ is both finite-to-one and well-founded.

(2) There is a Borel embedding of $f$ into $s_{[\mathbb{N}]}$.

(3) There is a Kakutani embedding of $f$ into $s_{[\mathbb{N}]}$.

By combining Theorems Jand $\mathrm{L}$, we obtain the following:

Theorem M. All aperiodic, essentially both finite-to-one and well-founded, non-smooth Borel functions on Polish spaces are Kakutani bi-reducible. Moreover, the class of such functions is closed under Kakutani equivalence.

Theorem N. All aperiodic Borel functions on Polish spaces which can be decomposed into an essentially injective, non-smooth part and an essentially both finite-to-one and well-founded, non-smooth part are Kakutani bi-reducible. Moreover, the class of such functions is closed under Kakutani equivalence.

In $\S 6$, we turn our attention to the class of antichainable, aperiodic Borel functions which are not essentially finite-to-one. Examples include the functions of the form $s_{\left[\omega^{\alpha}\right]}$, where $1<\alpha<\omega_{1}$ and the notation $\omega^{\alpha}$ refers to ordinal exponentiation. In fact, we show that every aperiodic, countable-to-one, well-founded Borel function is Borel embeddable into one of these. Although these functions are all Kakutani equivalent, it turns out that they are strictly $\leq_{K}$-increasing, thus Kakutani bi-reducibility is much finer than Kakutani equivalence.

We also consider functions whose restrictions to complete, stable Borel sets are illfounded. Examples include the restrictions $s_{\langle\alpha\rangle}$ of $s_{\alpha}$ to the sets of the form

$\langle\alpha\rangle^{\mathbb{N}}=\left\{x \in \alpha^{\mathbb{N}}: \forall n \in \mathbb{N}(x(n) \leq x(n+1))\right.$ and $\left.\forall n \in \mathbb{N} \exists m \geq n(x(m)<x(m+1))\right\}$.

Again, we see that the functions of the form $s_{\left\langle\omega^{\alpha}\right\rangle}$, where $1<\alpha<\omega_{1}$, are strictly $\leq_{K^{-}}$ increasing, and that every aperiodic, countable-to-one Borel function in a natural subclass of the antichainable functions is Borel embeddable into one of these.

The product of functions $f: X \rightarrow X$ and $g: Y \rightarrow Y$ is the function $f \times g: X \times Y \rightarrow$ $X \times Y$ given by $(f \times g)(x, y)=(f(x), g(y))$. We have thus far been unable to produce an aperiodic Borel function which lies strictly $\leq_{K}$-between $s_{[\mathbb{N}]}$ and $s_{[\mathbb{N}]} \times s_{(\mathbb{N})}$. In $\S 6$, we show that Kakutani equivalence of antichainable, aperiodic, countable-to-one Borel functions trivializes above the latter:

Theorem O. All antichainable, aperiodic, essentially countable-to-one Borel functions on Polish spaces to which $s_{[\mathbb{N}]} \times s_{(\mathbb{N})}$ is Kakutani reducible are Kakutani equivalent. Moreover, the class of such functions is closed under Kakutani bi-reducibility.

Theorem P. All aperiodic, essentially countable-to-one Borel functions on Polish spaces which can be decomposed into an antichainable part to which $s_{[\mathbb{N}]} \times s_{(\mathbb{N})}$ is Kakutani reducible and an essentially injective, non-smooth part are Kakutani equivalent. Moreover, the class of such functions is closed under Kakutani bi-reducibility. 
In $\S 7$, we consider the remaining gap in our knowledge of Kakutani equivalence of aperiodic, countable-to-one Borel functions:

Theorem Q. Suppose that $X$ is a Polish space and $f: X \rightarrow X$ is an aperiodic, essentially countable-to-one, non-smooth Borel function on a Polish space which is not Kakutani equivalent to one of the following functions:

(1) The odometer $\sigma$.

(2) The increasing shift $s_{[\mathbb{N}]}$.

(3) The disjoint sum $\sigma \oplus s_{[\mathbb{N}]}$.

(4) The 2-to-1 analog of the odometer $\sigma_{2}$.

(5) The injective shift $s_{(\mathbb{N})}$.

(6) The disjoint sum $\sigma \oplus s_{(\mathbb{N})}$.

Then $f$ can be decomposed into a part which is essentially injective and a part which is essentially strictly $\leq_{K}$-between $s_{[\mathbb{N}]}$ and $s_{[\mathbb{N}]} \times s_{(\mathbb{N})}$.

It is tempting to conjecture that there are no Borel functions which are strictly $\leq_{K^{-}}$ between $s_{[\mathbb{N}]}$ and $s_{[\mathbb{N}]} \times s_{(\mathbb{N})}$, from which it follows that Kakutani equivalence of aperiodic, countable-to-one Borel functions on uncountable Polish spaces is an equivalence relation with exactly seven classes (see Proposition 7.4). This conjecture, in turn, is a consequence of:

Conjecture R. Suppose that $f$ is an aperiodic, countable-to-one, well-founded Borel function on a Polish space. Then exactly one of the following holds:

(1) The function $f$ is essentially finite-to-one.

(2) There is a Kakutani reduction of $s_{[\mathbb{N}]} \times s_{(\mathbb{N})}$ to $f$.

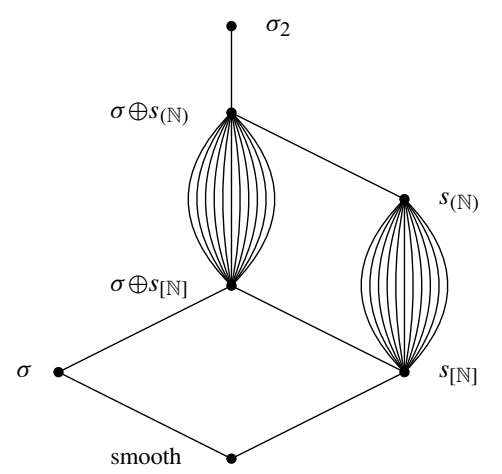

Fig. 1. Kakutani equivalence of countable-to-one Borel functions.

\subsection{Notation}

We gather here various notation for future reference. Suppose that $X$ and $Y$ are Polish spaces, $f: X \rightarrow X$, and $g: Y \rightarrow Y$. 
- The functions $f$ and $g$ are Borel isomorphic, or $f \cong_{B} g$, if there is a Borel isomorphism $\pi: X \rightarrow Y$ such that $\pi \circ f=g \circ \pi$.

- The function $f$ is Borel embeddable into the function $g$, or $f \sqsubseteq_{B} g$, if there is a stable Borel set $B \subseteq Y$ such that $f \cong_{B} g_{B}$.

- The function $f$ is Kakutani embeddable into the function $g$, or $f \sqsubseteq_{K} g$, if there is a recurrent Borel set $B \subseteq Y$ such that $f \cong_{B} g_{B}$.

- The functions $f$ and $g$ are Kakutani bi-embeddable, or $f \cong_{K} g$, if $f \sqsubseteq_{K} g$ and $g \sqsubseteq_{K} f$.

- The function $f$ is Kakutani reducible to the function $g$, or $f \leq_{K} g$, if there is a complete, stable Borel set $A \subseteq X$ such that $f_{A} \sqsubseteq K g$.

- The functions $f$ and $g$ are Kakutani bi-reducible, or $f \simeq_{K} g$, if $f \leq_{K} g$ and $g \leq_{K} f$.

- The function $f$ is Kakutani equivalent to the function $g$, or $f \approx_{K} g$, if there are complete, recurrent Borel sets $A \subseteq X$ and $B \subseteq Y$ such that $f_{A} \cong_{B} g_{B}$.

Clearly $\coprod_{B}$, $\subseteq_{K}$, and $\leq_{K}$ are quasi-orders, and $\cong_{B}, \simeq_{K}$, and $\simeq_{K}$ are equivalence relations (this remains open for $\approx_{K}$ ). They are related as follows: $f \cong_{B} g \Rightarrow f \cong_{K} g$ $\Rightarrow f \simeq_{K} g \Rightarrow f \approx_{K} g$ and $f \sqsubseteq_{B} g \Rightarrow f \sqsubseteq_{K} g \Rightarrow f \leq_{K} g$.

For each limit ordinal $\alpha<\omega_{1}$, the sets $[\alpha]^{\mathbb{N}}$ and $\langle\alpha\rangle^{\mathbb{N}}$ are given by

$$
[\alpha]^{\mathbb{N}}=\left\{x \in \alpha^{\mathbb{N}}: \forall n \in \mathbb{N}(x(n)<x(n+1))\right\}
$$

and

$\langle\alpha\rangle^{\mathbb{N}}=\left\{x \in \alpha^{\mathbb{N}}: \forall n \in \mathbb{N}(x(n) \leq x(n+1))\right.$ and $\left.\forall n \in \mathbb{N} \exists m \geq n(x(m)<x(m+1))\right\}$.

We use $s_{\alpha}$ to denote the unilateral shift on $\alpha^{\mathbb{N}}$, and we use $s_{[\alpha]}$ and $s_{\langle\alpha\rangle}$ to denote the restrictions of $s_{\alpha}$ to $[\alpha]^{\mathbb{N}}$ and $\langle\alpha\rangle^{\mathbb{N}}$. We also use $s_{\mathbb{N}}$ and $s_{[\mathbb{N}]}$ to denote $s_{\omega}$ and $s_{[\omega]}$, and we use $s_{(\mathbb{N})}$ to denote the restriction of $s_{\mathbb{N}}$ to the set $(\mathbb{N})^{\mathbb{N}}$ of injective sequences of natural numbers.

\section{Kakutani equivalence of automorphisms and $\mathbb{R}$-flows}

In this section, we completely classify aperiodic Borel automorphisms and $\mathbb{R}$-flows up to Kakutani equivalence and time-change isomorphism.

We first mention some basic facts concerning smooth functions. Suppose that $f$ : $X \rightarrow X$ and $A \subseteq X$. The forward ( $f$-) saturation of $A$ is given by $[A]_{f}=\bigcup_{n \in \mathbb{N}} f^{n}(A)$, and the $\left(f\right.$-)saturation of $A$ is given by $[A]_{f}=\bigcup_{n \in \mathbb{N}} f^{-n}\left([A]_{f}\right)$. If $A$ is a recurrent Borel set, then $[A]_{f}=\bigcup_{n \in \mathbb{N}} f^{-n}(A)$, thus $[A]_{f}$ is also Borel. We say that $A$ is $\left(f\right.$-)invariant if $A=f^{-1}(A)$, or equivalently, if $A=[A]_{f}$.

Proposition 2.1. Suppose that $X$ is a Polish space, $f: X \rightarrow X$ is a smooth Borel function, and $B \subseteq X$ is a recurrent Borel set. Then $f_{B}$ is smooth.

Proof. A partial ( $f$-)transversal is a set which intersects every orbit in at most one point. Note that if $A$ is a Borel partial transversal, then the non-negative powers of $f$ are injective on $A$, so each of the sets $f^{n}(A)$ is Borel (see, for example, Theorem 15.1 of [11]), thus so too is $[A]_{f}$. 
Fix a Borel transversal $A$ of $f$. Then the sets $A_{n}=f^{n}(A) \cap B$ are Borel partial $f_{B}$-transversals whose union intersects every $f_{B}$-orbit. It follows that the set $\bigcup_{n \in \mathbb{N}} A_{n} \backslash$ $\bigcup_{m<n}\left[A_{m}\right]_{f}$ is a Borel transversal of $f_{B}$, thus $f_{B}$ is smooth.

Proposition 2.2. All aperiodic, smooth Borel functions on Polish spaces with the same number of orbits are Kakutani bi-reducible. Moreover, the class of aperiodic, smooth Borel functions on Polish spaces with a given number of orbits is closed under Kakutani equivalence.

Proof. Suppose that $X$ and $Y$ are Polish spaces and $f: X \rightarrow X$ and $g: Y \rightarrow Y$ are aperiodic, smooth Borel functions with the same number of orbits. Fix Borel transversals $A \subseteq X$ and $B \subseteq Y$ of $f$ and $g$. As $|A|=|B|$, there is a Borel isomorphism $\phi: A \rightarrow B$ (see, for example, Theorem 15.6 of [11]). For each $x \in[A]_{f}$, let $n(x)$ denote the unique natural number $n$ such that $x \in f^{n}(A)$, and define $\psi:[A]_{f} \rightarrow A$ by $\psi(x)=y \Leftrightarrow$ $x=f^{n(x)}(y)$. Clearly $\psi$ is Borel (see, for example, Theorem 14.12 of [11]), so the map $\pi:[A]_{f} \rightarrow Y$ given by $\pi(x)=g^{n(x)} \circ \phi \circ \psi(x)$ is a Borel isomorphism of $f \mid[A]_{f}$ and $g \mid[B]_{g}$, thus $f \simeq_{K} g$.

Suppose now that $f$ and $g$ are Kakutani equivalent aperiodic Borel functions on Polish spaces. It is clear that $f$ and $g$ have the same number of orbits. Fix complete, recurrent Borel sets $A$ and $B$ such that $f_{A} \cong_{B} g_{B}$. Fix a Borel isomorphism $\pi$ of $f_{A}$ and $g_{B}$. If $g$ is smooth, then Proposition 2.1 ensures that there is a Borel $g_{B}$-transversal $C$. Then $\pi^{-1}(C)$ is a Borel $f$-transversal, thus $f$ is smooth.

Remark 2.3. A similar argument shows that if $f$ and $g$ are aperiodic, smooth Borel automorphisms of Polish spaces with the same number of orbits, then $f \cong_{B} g$.

Of course, the difficult part of our task is to understand Kakutani embeddability of non-smooth Borel automorphisms. A measure on $X$ is $(f$-)ergodic if every invariant Borel set is null or conull. Shelah-Weiss [18] have shown that every non-smooth Borel automorphism has an atomless, ergodic probability measure. The technical result from which their theorem was obtained can be rephrased as follows:

Theorem 2.4 (Shelah-Weiss). The odometer is continuously Kakutani embeddable into every non-smooth Borel automorphism of a Polish space.

That is, the odometer is $\sqsubseteq_{K}$-minimal among all non-smooth Borel automorphisms. By modifying the construction from the proof of Theorem 7.1 of [3], we have shown that the odometer is also maximal. Rather than give our original argument here, we will show instead a topological strengthening which has been subsequently obtained by BoykinJackson (a sketch of their proof appears in [1]).

Remark 2.5. Given a Polish space $X$ and a non-smooth Borel function $f: X \rightarrow X$, it will sometimes be useful to isolate an invariant Borel set $B \subseteq X$ on which $f$ is smooth and has uncountably many orbits. Although Silver's theorem [19] implies that this can be done, it seems worth noting that we can also obtain the desired fact as a corollary of the observation that there is such a set for the odometer, along with Theorems 2.4 
and 5.5 Moreover, in the special case that $f$ is injective, the latter theorem is unnecessary. Alternatively, in the special case that $f$ is countable-to-one (which is all that we require), the desired fact can also be obtained from a fairly straightforward splitting construction.

Suppose that $X$ and $Y$ are Polish spaces and $E$ and $F$ are Borel equivalence relations on $X$ and $Y$. A reduction of $E$ to $F$ is a function $\pi: X \rightarrow Y$ such that $x_{1} E x_{2} \Leftrightarrow$ $\pi\left(x_{1}\right) F \pi\left(x_{2}\right)$, for all $x_{1}, x_{2} \in X$. An embedding is an injective reduction. The tail equivalence relation induced by a Borel function $f: X \rightarrow X$ is given by

$$
x E_{t}(f) y \Leftrightarrow \exists m, n \in \mathbb{N}\left(f^{m}(x)=f^{n}(y)\right) .
$$

When $f$ is bijective, this is the usual orbit equivalence relation of $f$. The aperiodic part of $f$ is given by Aper $(f)=\left\{x \in X:\left|[x]_{f}\right|=\aleph_{0}\right\}$. It is straightforward to check that Aper $(f)$ is Borel and $f \mid(X \backslash \operatorname{Aper}(f))$ is smooth.

Theorem 2.6 (Boykin-Jackson). Suppose that $X$ is a zero-dimensional Polish space and $f: X \rightarrow X$ is a homeomorphism. Then there is a continuous embedding of $E_{t}(f)$ into $E_{t}(\sigma)$ whose restriction to the aperiodic part of $f$ is a Kakutani embedding.

Proof. Fix an enumeration $\left\langle U_{j}\right\rangle_{j \in \mathbb{N}}$ of an algebra of clopen sets which separates points of $X$, and set $U_{i j}=U_{j} \backslash \bigcup_{0<k<2^{i+1}} f^{-k}\left(U_{j}\right)$. Put $V_{i 0}=W_{i 0}=\emptyset$, and given $V_{i j}$ and $V_{i^{\prime}(j+1)}$, for $i^{\prime}<i$, define

$$
\begin{aligned}
W_{i(j+1)} & =\bigcup_{0<k<2^{i+1}} f^{-k}\left(V_{i j}\right) \cup \bigcup_{0<k<2^{i+1}} f^{k}\left(V_{i j}\right) \cup \bigcup_{i^{\prime}<i} V_{i^{\prime}(j+1)}, \\
V_{i(j+1)} & =V_{i j} \cup\left(U_{i j} \backslash W_{i(j+1)}\right) .
\end{aligned}
$$

It is clear that $V_{i j} \subseteq V_{i(j+1)}$ for all $i, j \in \mathbb{N}$, and this easily implies that $W_{i j} \subseteq W_{i(j+1)}$ for all $i, j \in \mathbb{N}$. It is also clear that for all $j \in \mathbb{N}$, the sets $V_{i j}$ are pairwise disjoint, thus so too are the sets $V_{i}=\bigcup_{j \in \mathbb{N}} V_{i j}$. Define $W_{i}=\bigcup_{j \in \mathbb{N}} W_{i j}$.

We say that a set $B \subseteq X$ is $k$-discrete if $f^{i}(B) \cap f^{j}(B)=\emptyset$ for all $i<j<k$.

Lemma 2.7. The set $V_{i}$ is a maximal $2^{i+1}$-discrete subset of $X \backslash W_{i}$.

Proof. A straightforward induction using the facts that $U_{i j}$ is $2^{i+1}$-discrete and $\bigcup_{0<k<2^{i+1}} f^{-k}\left(V_{i j}\right) \cup \bigcup_{0<k<2^{i+1}} f^{k}\left(V_{i j}\right) \subseteq W_{i(j+1)}$ shows that $V_{i j}$ is $2^{i+1}$-discrete, thus so too is $V_{i}$. Another straightforward induction using this fact then shows that $V_{i j} \cap W_{i j}=\emptyset$, thus $V_{i} \cap W_{i}=\varnothing$. To see that $V_{i}$ is maximal, suppose that $V_{i} \cup\{x\}$ is a $2^{i+1}$-discrete subset of $X \backslash W_{i}$, fix $j \in \mathbb{N}$ such that $\{x\}=U_{j} \cap\left\{f^{k}(x)\right\}_{k<2^{i+1}}$, and observe that $x \in U_{i j} \backslash W_{i} \subseteq U_{i j} \backslash W_{i(j+1)} \subseteq V_{i(j+1)} \subseteq V_{i}$.

Lemma 2.8. Suppose that $\left|[x]_{f}\right| \geq 2^{i+1}$. Then $x \in V_{i} \cup W_{i}$ and there exists $0<k<$ $3 \cdot 2^{i+1}$ such that $f^{-k}(x) \in V_{i}$.

Proof. To see that $x \in V_{i} \cup W_{i}$, fix $j \in \mathbb{N}$ such that $\{x\}=U_{j} \cap\left\{f^{k}(x)\right\}_{k<2^{i+1}}$, and observe that $x \in U_{i j}$, thus $x \in V_{i(j+1)} \cup W_{i(j+1)} \subseteq V_{i} \cup W_{i}$.

Now set $I=\left\{f^{-l}(x): 2^{i+1} \leq l<2 \cdot 2^{i+1}\right\}$. Lemma 2.7 implies that $\left|I \cap V_{i^{\prime}}\right| \leq$ $2^{i+1} / 2^{i^{\prime}+1}=2^{i-i^{\prime}}$ for all $i^{\prime} \leq i$, so $\left|I \cap \bigcup_{i^{\prime}<i} V_{i^{\prime}}\right| \leq \sum_{i^{\prime}<i} 2^{i-i^{\prime}}=\sum_{1 \leq i^{\prime} \leq i} 2^{i^{\prime}}<2^{i+1}$, 
thus there exists $2^{i+1} \leq l<2 \cdot 2^{i+1}$ such that $f^{-l}(x) \notin \bigcup_{i^{\prime}<i} V_{i^{\prime}}$. By Lemma 2.7, there exists $m<2^{i+1}$ such that $f^{-(l \pm m)}(x) \in V_{i}$, so $k=l \pm m$ is as desired.

For $i, j \in \mathbb{N}$ and $k<3 \cdot 2^{i+1}$, define $V_{i j k} \subseteq V_{i j}$ by

$$
V_{i j k}=\left\{x \in V_{i j}: \forall 0<i<k\left(f^{i}(x) \in W_{i j}\right) \text { and } f^{k}(x) \in V_{i j}\right\} .
$$

For $l<k$, put $X_{i j k l}=f^{l}\left(V_{i j k}\right)$. Set $X_{i j k}=\bigcup_{l<k} X_{i j k l}$ and $X_{i j}=\bigcup_{k<3 \cdot 2^{i+1}} X_{i j k}$, and for $x \in X_{i j}$, let $k_{i j}(x)$ and $l_{i j}(x)$ denote the unique natural numbers such that $x \in$

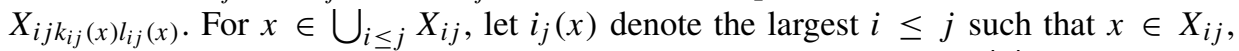
and set $d_{j}(x)=l_{i_{j}(x) j}(x)$. We also put $d_{j}(x)=i_{j}(x)=0$ for $x \notin \bigcup_{i \leq j} X_{i j}$. Define $f_{j}: X \rightarrow X$ by $f_{j}(x)=f^{-d_{j}(x)}(x)$.

Lemma 2.9. Suppose that $n \in \mathbb{N}$ and $x \in X$. Then there exists $j_{0} \in \mathbb{N}$ such that $f_{j}(x)=$ $f_{j} \circ f^{n}(x)$ for all $j \geq j_{0}$.

Proof. Suppose first that $[x]_{f}$ is finite. Clearly we can assume that $\left|[x]_{f}\right| \geq 2$, in which case Lemma 2.7 ensures that there is a largest $i \in \mathbb{N}$ such that $V_{i} \cap[x]_{f} \neq \emptyset$. Then $\left|V_{i} \cap[x]_{f}\right|=1$, since otherwise Lemma 2.7 implies that $\left|[x]_{f}\right| \geq 2^{i+2}$, and Lemma 2.8 then ensures that $V_{i+1} \cap[x]_{f} \neq \varnothing$, which contradicts our choice of $i$. Lemma 2.7 implies that $\left|[x]_{f}\right| \geq 2^{i+1}$, so Lemma 2.8 ensures that $[x]_{f} \subseteq V_{i} \cup W_{i}$. Fix $j_{0} \in \mathbb{N}$ such that $[x]_{f} \subseteq V_{i j_{0}} \cup W_{i j_{0}}$, and observe that if $j \geq j_{0}$, then $[x]_{f} \subseteq X_{i j}$, so $i_{j} \mid[x]_{f}$ is constant with value $i$, thus $f_{j} \mid[x]_{f}$ is constant with value the unique point of $V_{i} \cap[x]_{f}$. In particular, it follows that $f_{j}(x)=f_{j} \circ f^{n}(x)$.

Suppose now that $[x]_{f}$ is infinite. As the sets $V_{i}$ are pairwise disjoint, there exists $i_{0} \in \mathbb{N}$ such that $x, f(x), \ldots, f^{n}(x) \notin \bigcup_{i \geq i_{0}} V_{i}$. By Lemma 2.8 there exists $k<$ $3 \cdot 2^{i_{0}+1}-n$ least such that $f^{-k}(x) \in V_{i_{0}}$. As Lemma 2.8 also ensures that $[x]_{f} \subseteq V_{i_{0}} \cup W_{i_{0}}$, there exists $j_{0} \in \mathbb{N}$ such that $f^{l-k}(x) \in V_{i_{0} j_{0}} \cup W_{i_{0} j_{0}}$ for all $l<3 \cdot 2^{i_{0}+1}$. If $j \geq j_{0}$, then $i_{j}(x), i_{j} \circ f^{n}(x) \geq i_{0}$, so $i_{j}(x)=i_{j} \circ f^{n}(x)$, thus $f_{j}(x)=f_{j} \circ f^{n}(x)$.

We are now ready to describe the embedding. Let $x(i)$ denote $\chi_{U_{i}}(x)$, and let $x \mid k$ denote $\bigoplus_{i<k} x(i)$, where $\bigoplus$ denotes concatenation (and we abuse notation by identifying sequences of length one with their single entry).

Define $\phi_{j}: X \rightarrow 2^{j\left(3 \cdot 2^{j+1}\right)}$ by $\phi_{j}(x)=\bigoplus_{k<3 \cdot 2^{j+1}} f^{k} \circ f_{j}(x) \mid j$. The strings $\phi_{j}(x)$ simply code up larger and larger pieces of $[x]_{f}$. Note, however, that $[x]_{f}$ cannot be recovered from these pieces alone, as the relevant offsets are also required. These offsets are coded up by the maps $\psi_{j}: X \rightarrow 2^{j+5}$ given by

$$
\psi_{j}(x)=b\left(d_{j+1}(x)-d_{j}(x)+3 \cdot 2^{j+1}\right) \mid(j+5),
$$

where $b(i)=\sigma^{i}\left(0^{\infty}\right)$ denotes the reverse base 2 representation of $i$. We claim that the map $\pi: X \rightarrow 2^{\mathbb{N}}$ given by $\pi(x)=\bigoplus_{j \in \mathbb{N}} \phi_{j}(x) \psi_{j}(x)$ is as desired. Before we check this, it will be convenient to describe first $\leq_{\sigma}$ in a somewhat different fashion.

The reverse lexicographic ordering of $2^{n}$ is given by

$$
s \leq_{0} t \Leftrightarrow(s=t \text { or } s \circ \delta(s, t)<t \circ \delta(s, t)),
$$




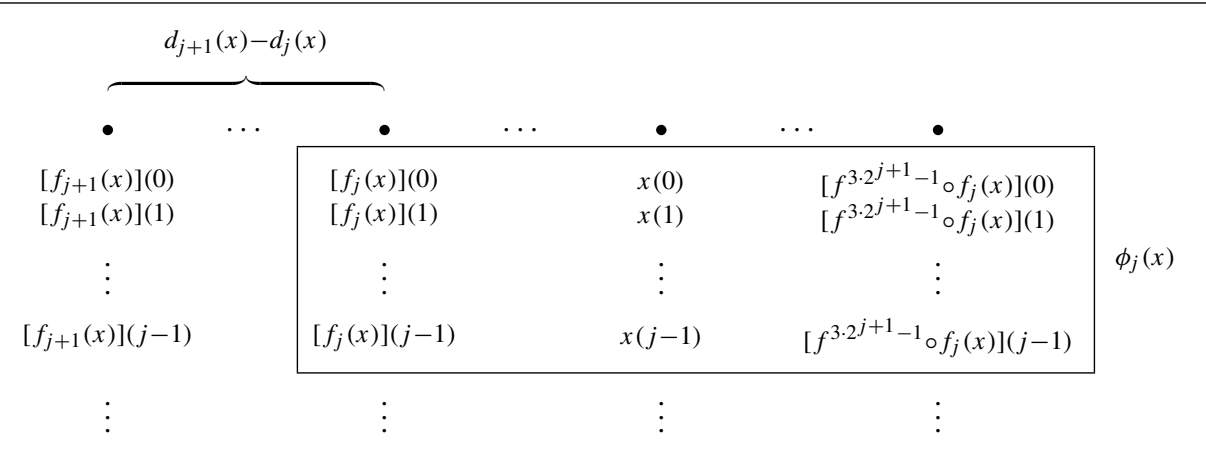

Fig. 2. $\phi_{j}(x)$ approximates $[x]_{f}$ and $\psi_{j}(x)$ codes the offset.

where $\delta(s, t)$ is the largest $m<n$ for which $s(m) \neq t(m)$. We define $\leq_{0}$ on $2^{\mathbb{N}}$ by

$$
x \leq_{0} y \Leftrightarrow \exists n \in \mathbb{N}\left(x\left|n \leq_{0} y\right| n \text { and } \forall m \geq n(x(m)=y(m))\right) .
$$

Define also $E_{0}$ on $2^{\mathbb{N}}$ by $x E_{0} y \Leftrightarrow \exists n \in \mathbb{N} \forall m \geq n(x(m)=y(m))$. A straightforward induction shows that $\left(E_{0}, \leq_{0}\right)$ and $\left(E_{t}(\sigma), \leq_{\sigma}\right)$ agree off the eventually constant sequences, and a casual inspection of the construction of $\pi$ reveals that no point of its range is eventually constant, so it only remains to show:

(1) If $x E_{t}(f) y$, then $\pi(x) E_{0} \pi(y)$.

(2) If $x<_{f} y$, then $\pi(x)<_{0} \pi(y)$.

(3) If $\pi(x) E_{0} \pi(y)$, then $x E_{t}(f) y$.

To see (1), appeal to Lemma 2.9 to find $j_{0} \in \mathbb{N}$ sufficiently large that $f_{j}(x)=f_{j}(y)$ for all $j \geq j_{0}$, and observe that $\phi_{j}(x)=\phi_{j}(y)$ and $\psi_{j}(x)=\psi_{j}(y)$, for all $j \geq j_{0}$, thus $\pi(x) E_{0} \pi(y)$.

To see (2), appeal to Lemma 2.9 to find $j_{0} \in \mathbb{N}$ least such that $f_{j}(x)=f_{j}(y)$ for all $j \geq j_{0}$. Then $j_{0}>0$ and $f^{-d_{j_{0}-1}(x)}(x)<_{f} f^{-d_{j_{0}-1}(y)}(y)$, so $d_{j_{0}}(x)-d_{j_{0}-1}(x)<$ $d_{j_{0}}(y)-d_{j_{0}-1}(y)$, thus $\psi_{j_{0}-1}(x)<_{0} \psi_{j_{0}-1}(y)$. As $\phi_{j}(x)=\phi_{j}(y)$ and $\psi_{j}(x)=\psi_{j}(y)$, for all $j \geq j_{0}$, it follows that $\pi(x)<_{0} \pi(y)$.

To see (3), suppose that $\phi_{j}(x)=\phi_{j}(y)$ and $\psi_{j}(x)=\psi_{j}(y)$, for all $j \geq j_{0}$, and set $i=d_{j_{0}}(x)-d_{j_{0}}(y)$. A straightforward induction shows that $i=d_{j}(x)-d_{j}(y)$ for all $j \geq j_{0}$. Identifying $\phi_{j}(x)$ and $\phi_{j}(y)$ with the corresponding elements of $\left(2^{j}\right)^{3 \cdot 2^{j+1}}$, it follows that if $j \geq j_{0}$, then

$$
\begin{aligned}
x \mid j & =f^{d_{j}(x)} \circ f_{j}(x)\left|j=\left[\phi_{j}(x)\right]\left(d_{j}(x)\right)=\left[\phi_{j}(y)\right]\left(d_{j}(y)+i\right)=f^{d_{j}(y)+i} \circ f_{j}(y)\right| j \\
& =f^{i}(y) \mid j
\end{aligned}
$$

so $x=f^{i}(y)$, thus $x E_{t}(f) y$.

By combining Theorems 2.4 and 2.6 we obtain a still stronger result: 
Theorem 2.10 (Boykin-Jackson). Suppose that $X$ and $Y$ are Polish spaces, $X$ is zerodimensional, $f: X \rightarrow X$ is a homeomorphism, and $g: Y \rightarrow Y$ is a non-smooth Borel automorphism. Then there is a continuous embedding of $E_{t}(f)$ into $E_{t}(g)$ whose restriction to the aperiodic part of $f$ is a Kakutani embedding.

Proof. The most natural proof of Theorem 2.4 first produces a continuous function $\phi$ : $2^{\mathbb{N}} \rightarrow Y$ such that $x \leq_{0} y \Leftrightarrow \phi(x) \leq_{g} \phi(y)$, for all $x, y \in 2^{\mathbb{N}}$ (in $\S 3$, we shall see how to accomplish such tasks in a somewhat more general setting). As the image of the embedding $\psi: X \rightarrow 2^{\mathbb{N}}$ produced by the proof of Theorem 2.6 avoids the eventually constant sequences, it follows that the map $\pi=\phi \circ \psi$ is as desired.

As a corollary, we now have the following fact (which we obtained some time before the more detailed analysis of Boykin-Jackson [1] was known):

Theorem 2.11. There are exactly two Kakutani bi-embeddability classes of aperiodic Borel automorphisms of uncountable Polish spaces. In order of Kakutani embeddability, these are:

(1) The aperiodic, smooth Borel automorphisms.

(2) The aperiodic, non-smooth Borel automorphisms.

Proof. It is enough to show that if $X$ and $Y$ are Polish spaces, $f: X \rightarrow X$ is an aperiodic Borel automorphism, and $g: Y \rightarrow Y$ is a non-smooth Borel automorphism, then $f \sqsubseteq_{K} g$. By standard change of topology results (see, for example, $\S 13$ of [11]), we can assume that $X$ is zero-dimensional and $f$ is a homeomorphism, and Theorem 2.10 then ensures that $f \sqsubseteq K g$.

This brings us to the primary result of this section:

Theorem 2.12. Up to Kakutani equivalence, there are exactly two aperiodic Borel automorphisms of uncountable Polish spaces.

Proof. We begin with the following general fact:

Lemma 2.13. Suppose that $X$ and $Y$ are Polish spaces, $f: X \rightarrow X$ and $g: Y \rightarrow Y$ are Borel functions, and $A \subseteq X$ and $B \subseteq Y$ are complete, recurrent Borel sets such that $f_{A} \sqsubseteq_{K} g$ and $g_{B} \sqsubseteq_{K} f$. Then $f \approx_{K} g$.

Proof. Fix Kakutani embeddings $\phi$ of $f_{A}$ into $g$ and $\psi$ of $g_{B}$ into $f$. We proceed via a standard Schröder-Bernstein argument.

Set $C_{0}=X \backslash[\psi(B)]_{f}$, and recursively define $D_{n}=\left[\phi\left(A \cap C_{n}\right)\right]_{g}$ and $C_{n+1}=$ $\left[\psi\left(B \cap D_{n}\right)\right]_{f}$. Setting $C=\bigcup_{n \in \mathbb{N}} C_{n}$ and $D=\bigcup_{n \in \mathbb{N}} D_{n}$, it follows that $\phi$ is a Kakutani embedding of $f_{A \cap C}$ onto a $g_{D}$-complete set.

To see that $\psi$ is a Kakutani embedding of $g_{B \backslash D}$ onto an $f_{X \backslash C}$-complete set, observe that $[\psi(B \backslash D)]_{f}=[\psi(B)]_{f} \backslash[\psi(B \cap D)]_{f}$, since $\psi$ is a Kakutani embedding and $D$ is $g$-invariant, and

$$
[\psi(B)]_{f} \backslash \bigcup_{n \in \mathbb{N}}\left[\psi\left(B \cap D_{n}\right)\right]_{f}=\left(X \backslash C_{0}\right) \backslash \bigcup_{n \in \mathbb{N}} C_{n+1}=X \backslash C .
$$




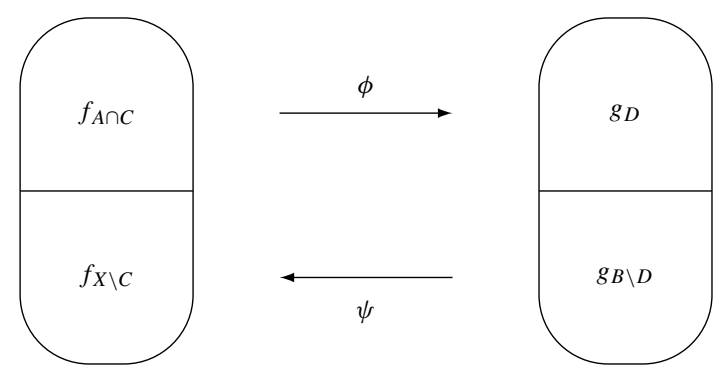

Fig. 3. A witness to the Kakutani equivalence of $f$ and $g$.

Set $C^{\prime}=(A \cap C) \cup \psi(B \backslash D)$ and $D^{\prime}=\phi(A \cap C) \cup(B \backslash D)$, and observe that $\phi\left|(A \cap C) \oplus \psi^{-1}\right| \psi(B \backslash D)$ is a Borel isomorphism of $f_{C^{\prime}}$ and $g_{D^{\prime}}$, thus $f \approx_{K} g$.

The desired result clearly follows from Theorem 2.11 and Lemma 2.13

We can describe also the Kakutani equivalence class of the non-smooth Borel automorphisms within the class of all aperiodic Borel functions:

Theorem 2.14. All aperiodic, essentially injective, non-smooth Borel functions on Polish spaces are Kakutani bi-reducible. Moreover, the class of such functions is closed under Kakutani equivalence.

Proof. Suppose first that $X$ and $Y$ are Polish spaces and $f: X \rightarrow X$ and $g: Y \rightarrow Y$ are aperiodic, essentially injective, non-smooth Borel functions. Fix a complete, recurrent Borel set $A \subseteq X$ such that $f_{A}$ is injective. A straightforward induction shows that $\bigcup_{i \leq n} f^{i}(A)$ is a recurrent Borel set whose induced transformation is injective, thus $[A]_{f} \vec{f}$ is a stable Borel set whose induced transformation is injective. By replacing $A$ with its forward saturation, we can therefore assume that $A$ is stable. Similarly, there is a complete, stable Borel set $B \subseteq Y$ such that $g_{B}$ is injective. By throwing out invariant Borel sets on which $f$ and $g$ are smooth and have uncountably many orbits, we can assume that $f_{A}$ is a Borel automorphism of $A$ and $g_{B}$ is a Borel automorphism of $B$. Then $f_{A} \cong_{K} g_{B}$, by Theorem 2.11

Suppose now that $f$ and $g$ are Kakutani equivalent aperiodic Borel functions on Polish spaces. If $g$ is non-smooth, then Proposition 2.2 ensures that $f$ is non-smooth. If $g$ is essentially injective, then there is a $g$-complete, stable Borel set whose induced transformation is injective. As such sets can be pulled back through Kakutani embeddings, it follows that $f$ is essentially injective.

In light of the proof of Theorem 2.12, it is natural to ask, when given two aperiodic, non-smooth Borel automorphisms, whether there is necessarily a Kakutani embedding of one onto a complete set of the other. Dougherty-Jackson-Kechris [3] have shown the weakening of Theorem 2.11 where Kakutani embeddability of $f$ into $g$ is replaced with Borel embeddability of $E_{t}(f)$ into $E_{t}(g)$. In fact, the analogous question for Borel embeddability has a positive answer: 
Proposition 2.15. Suppose that $f$ and $g$ are aperiodic, countable-to-one, non-smooth Borel functions on Polish spaces. Then at least one of $E_{t}(f)$ or $E_{t}(g)$ is Borel embeddable onto a complete set of the other.

Proof. Suppose that $X$ is a Polish space and $E$ is a countable Borel equivalence relation on $X$. The (E-)saturation of a set $B \subseteq X$ is given by $[B]_{E}=\{x \in X: \exists y \in B(x E y)\}$. The Lusin-Novikov uniformization theorem (see, for example, Theorem 18.10 of [11]) implies that saturations of Borel sets are Borel. A set $B \subseteq X$ is (E-)invariant if $B=$ $[B]_{E}$, and a measure on $X$ is $(E$-)ergodic if every invariant Borel subset of $X$ is null or conull. A measure on $X$ is (E-)invariant if every Borel automorphism of $X$ whose graph is contained in $E$ is measure preserving.

We use $\mathcal{E} \mathcal{I}(E)$ to denote the set of ergodic, invariant probability measures on $X$. The Farrell-Varadarajan uniform ergodic decomposition theorem [4, 20] ensures that $\mathcal{E} \mathcal{I}(E)$ is a Borel subset of the Polish space of all probability measures on $X$, thus $|\mathcal{E} \mathcal{I}(E)| \in$ $\left\{0,1, \ldots, \aleph_{0}, \mathfrak{c}\right\}$ (see, for example, Theorem 13.6 of [11]).

A transversal of $E$ is a set which intersects every $E$-class in exactly one point, and a set is (E-)complete if it intersects every $E$-class in at least one point. We say that $E$ is smooth if it has a Borel transversal, and we say that $E$ is aperiodic if all of its equivalence classes are infinite.

Lemma 2.16. Suppose that $X$ is a Polish space, $E$ is an aperiodic, non-smooth countable Borel equivalence relation on $X,|\mathcal{E} \mathcal{I}(E)|=\kappa$, and $\lambda \in\left\{0,1, \ldots, \aleph_{0}, \mathfrak{c}\right\}$. Then there is a complete Borel set $D \subseteq X$ such that $|\mathcal{E} \mathcal{I}(E \mid D)|=\kappa+\lambda$ and $E \mid D$ is aperiodic.

Proof. By the Harrington-Kechris-Louveau theorem [8], there is a continuous embedding $\phi$ of $E_{0}$ into $E$. Define $\psi: 2^{\mathbb{N}} \times 2^{\mathbb{N}} \rightarrow 2^{\mathbb{N}}$ by $\psi(x, y)=\bigoplus_{n \in \mathbb{N}}(x \mid n) \oplus y(n)$, and observe that for all $x \in 2^{\mathbb{N}}$, the function $\psi_{x}: 2^{\mathbb{N}} \rightarrow 2^{\mathbb{N}}$ given by $\psi_{x}(y)=\psi(x, y)$ is an embedding of $E_{0}$ into itself, thus the well known fact that the usual product measure on $2^{\mathbb{N}}$ is the unique ergodic, invariant probability measure for $E_{0}$ implies that its image under $\psi_{x}$ is the unique ergodic, invariant probability measure for $E_{0} \mid \psi_{x}\left(2^{\mathbb{N}}\right)$. Fix a Borel set $A \subseteq 2^{\mathbb{N}}$ of cardinality $\lambda$, and observe that the restriction of $E_{0}$ to the set $B=\psi\left(A \times 2^{\mathbb{N}}\right)$ has $\lambda$-many ergodic, invariant probability measures (since the saturations of the sets of the form $\psi_{x}\left(2^{\mathbb{N}}\right)$ are pairwise disjoint), while the restriction of $E_{0}$ to $[B]_{E_{0}}$ has none (since the latter set is null with respect to the usual product measure). Set $C=X \backslash[\phi(B)]_{E}$ and $D=C \cup \phi(B)$. Then $|\mathcal{E} \mathcal{I}(E \mid D)|=|\mathcal{E} \mathcal{I}(E \mid C)|+|\mathcal{E} \mathcal{I}(E \mid \phi(B))|=$ $|\mathcal{E} \mathcal{I}(E)|+\left|\mathcal{E} \mathcal{I}\left(E_{0} \mid B\right)\right|=\kappa+\lambda$.

By reversing the roles of $f$ and $g$ if necessary, we can assume that $E_{t}(f)$ admits at least as many ergodic, invariant probability measures as $E_{t}(g)$. By Lemma 2.16, there is a complete Borel set $B$ such that $E_{t}(g) \mid B$ is aperiodic and $E_{t}(f)$ and $E_{t}(g) \mid B$ admit the same number of ergodic, invariant probability measures. It then follows from Corollary 8.2 and Theorem 9.1 of [3] that $E_{t}(f)$ is Borel isomorphic to $E_{t}(g) \mid B$, and any such isomorphism gives rise to the desired embedding.

Nevertheless, we have the following negative answer to our original question:

Proposition 2.17. There is a $\sigma$-invariant Borel set $B \subseteq 2^{\mathbb{N}}$ such that neither $\sigma \mid B$ nor $\sigma \mid\left(2^{\mathbb{N}} \backslash B\right)$ is Kakutani embeddable onto a complete set of the other. 
Proof. Let $\mu$ denote the usual product measure on $2^{\mathbb{N}}$, and note that since $\mu$ is the unique ergodic, invariant probability measure for $\sigma$, then for every $\sigma$-invariant Borel set $B \subseteq 2^{\mathbb{N}}$, exactly one of $\sigma \mid B$ and $\sigma \mid\left(2^{\mathbb{N}} \backslash B\right)$ admits an ergodic, invariant probability measure. We will arrange things so that $\sigma \mid B$ admits such a measure. This guarantees that $\sigma \mid\left(2^{\mathbb{N}} \backslash B\right)$ is not Kakutani embeddable onto a $(\sigma \mid B)$-complete set, since $\mu$ could be pulled back through any such embedding.

Lemma 1.17 of [9] ensures that for each $n \in \mathbb{Z}^{+}$, there is a maximal $\left(n \cdot 3^{n}\right)$-discrete Borel set $A_{n} \subseteq X$. Set $A_{n}^{\prime}=\bigcup_{i<n} \sigma^{i}\left(A_{n}\right)$, noting that $\mu\left(A_{n}^{\prime}\right) \leq 1 / 3^{n}$. Set $A=2^{\mathbb{N}} \backslash$ $\bigcup_{n \in \mathbb{Z}^{+}} A_{n}^{\prime}$ and $B=[A]_{\sigma}$. As $\mu(A) \geq 1 / 2$, it follows that $\mu(B)=1$.

Suppose, towards a contradiction, that $\pi: B \rightarrow 2^{\mathbb{N}} \backslash B$ is a Kakutani embedding of $\sigma \mid B$ onto a $\sigma \mid\left(2^{\mathbb{N}} \backslash B\right)$-complete set. Then the set $A^{\prime}=A \cup \pi(A)$ has large gaps on every orbit, in the sense that

$$
\forall x \in 2^{\mathbb{N}} \forall m \in \mathbb{N} \exists n \in \mathbb{N}\left(\sigma^{n}(x), \sigma^{n+1}(x), \ldots, \sigma^{n+m}(x) \notin A^{\prime}\right) .
$$

However, the fact that $A^{\prime}$ is complete ensures that there exist $n \in \mathbb{N}$ and $s \in 2^{n}$ such that $\mathcal{N}_{s} \backslash A^{\prime}$ is meager, thus so too is the set $\bigcup_{k \in \mathbb{Z}} \sigma^{k \cdot 2^{n}}\left(\mathcal{N}_{s} \backslash A^{\prime}\right)$. As $\sigma^{2^{n}}\left(\mathcal{N}_{s}\right)=\mathcal{N}_{s}$, it then follows that the set $\mathcal{N}_{s} \backslash \bigcap_{k \in \mathbb{Z}} \sigma^{k \cdot 2^{n}}\left(A^{\prime}\right)$ is meager, so there exists $x \in \mathcal{N}_{s}$ such that $\sigma^{k \cdot 2^{n}}(x) \in A^{\prime}$ for all $k \in \mathbb{Z}$, which contradicts $(\dagger)$.

Remark 2.18. In fact, there are large collections of Borel automorphisms whose induced equivalence relations are Borel isomorphic, but for which no automorphism in the collection is Kakutani embeddable onto a complete set of any of the others. This follows from the deep results of Ornstein-Rudolph-Weiss [16] on the usual measure-theoretic notion of Kakutani equivalence.

Next we take care of the second goal of this section:

Theorem 2.19. All free, non-smooth Borel $\mathbb{R}-$ flows on Polish spaces are time-change isomorphic.

Proof. Wagh [21] has shown that every free Borel $\mathbb{R}$-flow $X$ admits a complete Borel set $A \subseteq X$ which is $\leq_{X}$-discrete, in the sense that there exists $\epsilon>0$ such that $t+x \notin A$ for all $0<t<\epsilon$ and $x \in A$. Clearly such a set can be modified so as to ensure that its intersection with each orbit is of type $\mathbb{Z}$, in which case there is an aperiodic Borel automorphism $f: A \rightarrow A$ of the complete set which induces the same partial orderings of its orbits as does the flow. Suppose now that $Y$ is another free Borel $\mathbb{R}$-flow, and build a Borel complete set $B \subseteq Y$ and an aperiodic Borel automorphism $g: B \rightarrow B$ in the same fashion. If $X$ and $Y$ are non-smooth, then $f$ and $g$ are non-smooth, so Theorem 2.12 implies that $f \approx_{K} g$, from which it easily follows that $X$ and $Y$ are time-change isomorphic.

Remark 2.20. Suppose that $X$ and $Y$ are Borel $\mathbb{R}$-flows, and set $\phi_{r}(x)=r+x$. We say that $X$ and $Y$ are $C^{\infty}$-time-change isomorphic if there are Borel functions $\pi: X \rightarrow Y$ and $f: X \times \mathbb{R} \rightarrow \mathbb{R}$ such that, if we set $f_{x}(r)=f(x, r)$, then:

(1) $\forall x \in X$ ( $f_{x}$ is an increasing, $C^{\infty}$ function which fixes 0$)$.

(2) $\forall x_{1}, x_{2} \in X \forall r \in \mathbb{R}\left(x_{2}=\phi_{r}\left(x_{1}\right) \Leftrightarrow \pi\left(x_{2}\right)=\phi_{f_{x_{1}}(r)} \circ \pi\left(x_{1}\right)\right)$. 
It is not hard to modify the above argument to show that any two free, non-smooth Borel $\mathbb{R}$-flows are $C^{\infty}$-time-change isomorphic.

Remark 2.21. The notion of $C^{\infty}$-time-change isomorphism applies to all (not necessarily free) Borel $\mathbb{R}$-flows. Using a theorem of D. E. Miller [14], it is not difficult to classify all Borel $\mathbb{R}$-flows up to $C^{\infty}$-time-change isomorphism. Let $\operatorname{Fix}(X)$ denote the set of $x \in X$ for which $\left|[x]_{\mathbb{R}}\right|=1$, and let $\operatorname{Per}(X)$ denote the set of $x \in X \backslash \operatorname{Fix}(X)$ such that $\phi_{r}(x)=x$ for some $r>0$. The restriction of the $\mathbb{R}$-flow to $\operatorname{Fix}(X) \cup \operatorname{Per}(X)$ is smooth. Let $\operatorname{Aper}(X)=X \backslash(\operatorname{Fix}(X) \cup \operatorname{Per}(X))$. Then $X$ and $Y$ are $C^{\infty}$-time-change isomorphic if and only if $|\operatorname{Fix}(X)|=|\operatorname{Fix}(Y)|,|\operatorname{Per}(X)|=|\operatorname{Per}(Y)|,|\operatorname{Aper}(X)|=|\operatorname{Aper}(Y)|$, and $X$ is smooth $\Leftrightarrow Y$ is smooth.

Finally, we remark that the sorts of results we have obtained in this section break down if we substantially strengthen the notion of Kakutani equivalence. If we require, for example, that the images of our embeddings have bounded gaps, then there are continuum-sized collections of Borel automorphisms which are incomparable, and the notion of embeddability becomes $\Sigma_{2}^{1}$-complete (in the codes).

\section{Dichotomy theorems for generalizations of the odometer}

In this section, we prove Glimm-Effros-style dichotomy theorems which characterize the circumstances under which certain generalizations of the odometer are Kakutani embeddable into a given Borel function.

We first describe our generalizations of the odometer. For notational convenience, we make the usual identification of each natural number $d$ with the set of strictly smaller natural numbers. We also identify $d \times 2^{0}$ with $d$, and $\left(d \times 2^{n}\right) \times 2$ with $d \times 2^{n+1}$. A $d$-blueprint is a sequence $\left\langle s_{n}\right\rangle_{n \in \mathbb{N}} \in \prod_{n \in \mathbb{N}} d \times 2^{n}$ such that:

(1) $\forall m, n \in \mathbb{N}\left(s_{m} 1 \nsubseteq s_{n}\right)$.

(2) $\forall s \in d \times 2^{<\mathbb{N}} \exists n \in \mathbb{N}\left(s \sqsubseteq s_{n}\right.$ or $\left.s_{n} 1 \sqsubseteq s\right)$.

Given such a $d$-blueprint, set $D_{d, 0}=d$ and $R_{d, 0}=\{d\}$, and recursively define $D_{d, n}, R_{d, n}$ $\subseteq(d+1) \times 2^{n}$ by

$$
D_{d, n+1}=\left(D_{d, n} \times 2\right) \cup\left\{d 1^{n} 0\right\} \quad \text { and } \quad R_{d, n+1}=\left(R_{d, n} \times 2\right) \cup\left\{s_{n} 1\right\} .
$$

A straightforward induction ensures that $d 1^{n} 0 \notin D_{d, n} \times 2$ (and a straightforward induction using condition (1) ensures that $s_{n} 1 \notin R_{d, n} \times 2$ ). We can therefore recursively define $\sigma_{d, n}: D_{d, n} \rightarrow R_{d, n}$ by setting $\sigma_{d, 0}(c)=d$ and

$$
\sigma_{d, n+1}(s i)= \begin{cases}\sigma_{d, n}(s) i & \text { if } s \in D_{d, n} \\ s_{n} 1 & \text { if } s i=d 1^{n} 0\end{cases}
$$

Set $D_{d}=\bigcup_{n \in \mathbb{N}}\left\{s x: s \in D_{d, n}\right.$ and $\left.x \in 2^{\mathbb{N}}\right\}$ and $R_{d}=\bigcup_{n \in \mathbb{N}}\left\{s x: s \in R_{d, n}\right.$ and $\left.x \in 2^{\mathbb{N}}\right\}$, and define $\sigma_{d}: D_{d} \rightarrow R_{d}$ by $\sigma_{d}(s x)=\sigma_{d, n}(s) x$ for $n \in \mathbb{N}$ and $s \in D_{d, n}$. 


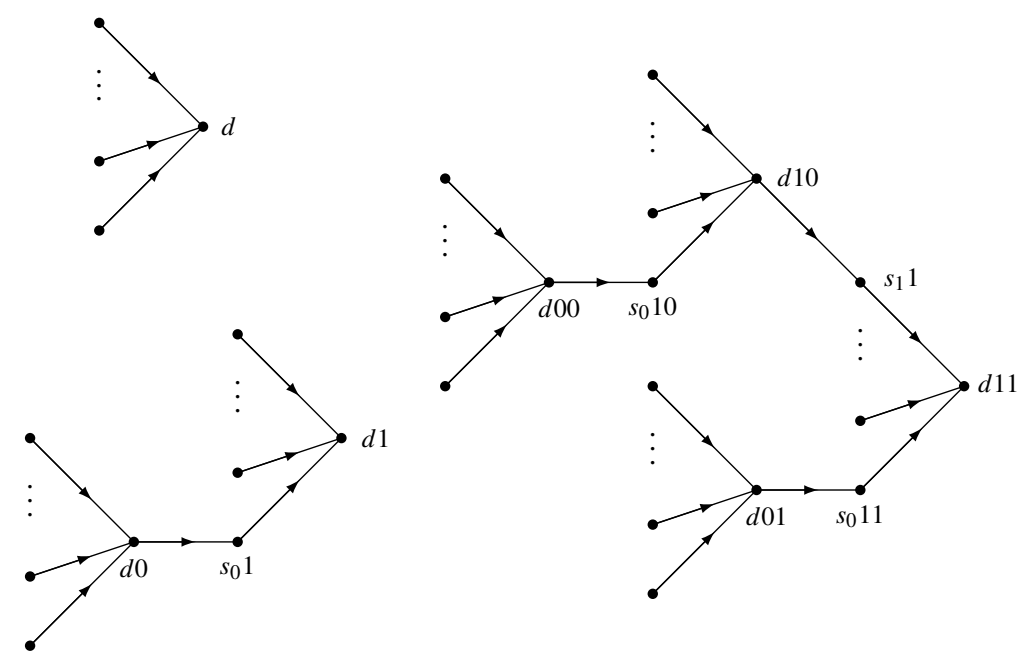

Fig. 4. The first three approximations $\sigma_{d, 0}, \sigma_{d, 1}$, and $\sigma_{d, 2}$ to $\sigma_{d}$.

Remark 3.1. A straightforward induction shows that the unique 1-blueprint is given by $s_{n}=0^{n+1}$. The corresponding function $\sigma_{1}$ is simply the restriction of the odometer to the set $2^{\mathbb{N}} \backslash\left\{1^{\infty}\right\}$.

Remark 3.2. For $d \geq 2$, there are many $d$-blueprints. Nevertheless, the results of this section imply that the exact choice of blueprint (or even the choice of $d \geq 2$ ) does not affect the Kakutani bi-embeddability class of $\sigma_{d}$. This is the reason we do not bother to include the $d$-blueprint we have in mind in our notation for $\sigma_{d}$.

We also use $E_{0}$ to denote the analogous equivalence relation on $(d+1) \times 2^{\mathbb{N}}$.

Proposition 3.3. The tail equivalence relation induced by $\sigma_{d}$ is $E_{0}$.

Proof. It is clear that $E_{t}\left(\sigma_{d}\right) \subseteq E_{0}$. To see that $E_{0} \subseteq E_{t}\left(\sigma_{d}\right)$, note first that by a straightforward induction, for each $n \in \mathbb{N}$ and $s, t \in(d+1) \times 2^{n}$ there exist $j, k \in \mathbb{N}$ such that $\sigma_{d, n}^{j}(s)=\sigma_{d, n}^{k}(t)=d 1^{n}$. Suppose now that $x E_{0} y$, and fix $n \in \mathbb{N}$ such that $x(m)=y(m)$ for all $m>n$, as well as $j, k \in \mathbb{N}$ such that $\sigma_{d, n}^{j}(x \mid(n+1))=\sigma_{d, n}^{k}(y \mid(n+1))$. Then $\sigma_{d}^{j}(x)=\sigma_{d}^{k}(y)$, thus $x E_{t}\left(\sigma_{d}\right) y$.

Proposition 3.4. Suppose that $B \subseteq(d+1) \times 2^{\mathbb{N}}$ is a non-meager Borel set. Then $B$ is not a $\sigma_{d}$-antichain.

Proof. Fix $n \in \mathbb{N}$ and $s \in(d+1) \times 2^{n}$ such that $B$ is comeager in $\mathcal{N}_{s}$. Fix $t \leq \sigma_{d, n} s$ which is not in $R_{d, n}$. Condition (2) ensures that there exist $m \geq n$ and $u \in 2^{m-n}$ such that $s_{m}=t u$. Then $s u 0 \leq \sigma_{d, m+1} d 1^{m} 0 \leq \sigma_{\sigma_{d, m+1}} t u 1 \leq \sigma_{d, m+1} s u 1$. Fix $x \in 2^{\mathbb{N}}$ with su $0 x$, su $1 x \in B$, and note that $s u 0 x \leq \sigma_{d} s u 1 x$, thus $B$ is not an antichain.

We say that $x$ and $y$ are $\left(f\right.$-)incomparable, or $x \perp_{f} y$, if $x \bigsqcup_{f} y$ and $y \not_{f} x$. We say that a set $A \subseteq X$ is $\left(f\right.$-) linear if $x \leq_{f} y$ or $y \leq_{f} x$, for all $x, y \in E_{t}(f) \mid A$. 
Proposition 3.5. Suppose that $d \geq 2$ and $B \subseteq(d+1) \times 2^{\mathbb{N}}$ is a non-meager Borel set. Then $B$ is not $\sigma_{d}$-linear.

Proof. Fix $n \in \mathbb{N}$ and $s \in(d+1) \times 2^{n}$ such that $B$ is comeager in $\mathcal{N}_{s}$. As $d \geq 2$, a straightforward induction shows that there exists $t \in D_{d, n} \backslash\left(R_{d, n} \cup\left\{s_{n}\right\}\right)$, and it follows that $t 1 \notin R_{d, n+1}$ and $s 0 \perp_{\sigma_{d, n+1}} t$. Condition (2) ensures that there exist $m>n$ and $u \in 2^{m-n-1}$ such that $s_{m}=t 1 u$. Then $s 0 u 0 \leq_{\sigma_{d, m+1}} d 1^{m} 0 \leq_{\sigma_{d, m+1}} t 1 u 1$. As $s 0 u 1 \perp_{\sigma_{d, m+1}} t 1 u 1$ and the forward orbit of $s 0 u 0$ is linearly ordered by $\leq_{\sigma_{d, m+1}}$, it follows that $s 0 u 0 \perp_{\sigma_{d, m+1}} s 0 u 1$. Fix $x \in 2^{\mathbb{N}}$ such that $s 0 u 0 x, s 0 u 1 x \in B$, and observe that $s 0 u 0 x \perp_{\sigma_{d}} s 0 u 1 x$, thus $B$ is not linear.

Given a partial function $f$ on $X$, we say that a set $A \subseteq X$ is $(f$-)recurrent if for all $x \in A \cap \operatorname{dom}(f)$, there exists $n \in \mathbb{Z}^{+}$such that $f^{n}(x) \in A$. We then define Kakutani embeddability and equivalence of Borel partial functions as before.

Given a partial function $p$ on a finite set $S$, we use $\operatorname{Kak}(p, f)$ to denote the set of all Kakutani embeddings of $p$ into $f$, and we use $\mathcal{I}_{p}$ to denote the $\sigma$-ideal generated by the sets of the form $\{\pi \in \operatorname{Kak}(p, f): \pi(s) \in B\}$, where $s \in S$ and $B$ is a Borel $f$-antichain. We say that $\mathcal{I}_{p}$ trivializes if $\operatorname{Kak}(p, f) \in \mathcal{I}_{p}$.

Theorem 3.6. Suppose that $X$ is a Polish space and $f: X \rightarrow X$ is a Borel function. Then exactly one of the following holds:

(1) The $\sigma$-ideal $\mathcal{I}_{\sigma_{d, 0}}$ trivializes.

(2) There is a continuous Kakutani embedding of $\sigma_{d}$ into $f$.

Proof. To see that (1) and (2) are mutually exclusive suppose, towards a contradiction, that $\mathcal{I}_{\sigma_{d, 0}}$ trivializes and there is a Kakutani embedding $\pi$ of $\sigma_{d}$ into $f$. Fix Borel antichains $A_{n} \subseteq X$ whose union intersects the range of every Kakutani embedding of $\sigma_{d, 0}$ into $f$, and put $B_{n}=\pi^{-1}\left(A_{n}\right)$. Then each of the sets $B_{n}$ is an antichain, and their union $B$ intersects the range of every Kakutani embedding of $\sigma_{d, 0}$ into $\sigma_{d}$. Proposition 3.4 ensures that $B$ is meager, thus so too is $[B]_{\sigma_{d}}$. Fix $x \in\left((d+1) \times 2^{\mathbb{N}}\right) \backslash[B]_{\sigma_{d}}$, and observe that $\sigma_{d, 0} \square_{K} \sigma_{d} \mid[x]_{\sigma_{d}}$, which is absurd.

It remains to show that if (1) fails, then (2) holds. By standard change of topology results, we can assume that $f$ is continuous. We also assume that $X=\mathbb{N}^{\mathbb{N}}$, as the general case is handled similarly. A $(d, n)$-configuration is a quadruple $(k, u, v, w)$, where $k \in \mathbb{N}$, $u:(d+1) \times 2^{n} \rightarrow \mathbb{N}^{k}, v: d \rightarrow \mathbb{Z}^{+}$, and $w: n \rightarrow \mathbb{Z}^{+}$, with the following properties:

- $\forall i, j \leq n \forall s, t \in(d+1) \times 2^{n}\left(s(n) \neq t(n) \Rightarrow f^{i}\left(\mathcal{N}_{u(s)}\right) \cap f^{j}\left(\mathcal{N}_{u(t)}\right)=\emptyset\right)$.

- $\forall i<n \forall s, t \in(d+1) \times 2^{n}\left(s \mathbb{\leq}_{\sigma_{d, n}} t \Rightarrow f^{i}\left(\mathcal{N}_{u(s)}\right) \cap \mathcal{N}_{u(t)}=\emptyset\right)$.

Associated with $(k, u, v, w)$ is the set $\operatorname{Kak}(k, u, v, w)$ of Kakutani embeddings $\pi$ : $(d+1) \times 2^{n} \rightarrow \mathbb{N}^{\mathbb{N}}$ of $\sigma_{d, n}$ into $f$ with the following properties:

- $\forall s \in(d+1) \times 2^{n}\left(\pi(s) \in \mathcal{N}_{u(s)}\right)$.

- $\forall c<d \forall s \in 2^{n}\left(\pi(d s)=f^{v(c)} \circ \pi(c s)\right)$.

- $\forall m<n \forall t \in 2^{n-m-1}\left(\pi\left(s_{m} 1 t\right)=f^{w(m)} \circ \pi\left(d 1^{m} 0 t\right)\right)$.

We say that $(k, u, v, w)$ is reasonable if $\operatorname{Kak}(k, u, v, w) \notin \mathcal{I}_{\sigma_{d, n}}$.

An extension of $(k, u, v, w)$ is a $(d, n+1)$-configuration $\left(k^{\prime}, u^{\prime}, v^{\prime}, w^{\prime}\right)$ such that $u(s) \sqsubseteq u^{\prime}(s i), v=v^{\prime}$, and $w=w^{\prime} \mid n$, for all $i<2$ and $s \in(d+1) \times 2^{n}$. 
Lemma 3.7. Every reasonable $(d, n)$-configuration has a reasonable extension.

Proof. Suppose, towards a contradiction, that $(k, u, v, w)$ is a reasonable $(d, n)$-configuration with no reasonable extension. Then there are countably many Borel antichains whose union $B$ intersects the range of every Kakutani embedding in $\operatorname{Kak}\left(k^{\prime}, u^{\prime}, v^{\prime}, w^{\prime}\right)$, for every extension $\left(k^{\prime}, u^{\prime}, v^{\prime}, w^{\prime}\right)$ of $(k, u, v, w)$. Define

$$
\mathcal{A}=\left\{\pi \in \operatorname{Kak}(k, u, v, w): \pi\left((d+1) \times 2^{n}\right) \subseteq \operatorname{Aper}(f) \backslash B\right\} .
$$

The fact that $(k, u, v, w)$ is reasonable ensures that $\mathcal{A} \notin \mathcal{I}_{\sigma_{d, n}}$.

Let $S$ denote the (necessarily finite) set of pairs $(i, s) \in \mathbb{N} \times\left((d+1) \times 2^{n}\right)$ such that $f^{i} \circ \pi(s) \leq_{f} f^{n} \circ \pi\left(d 1^{n}\right)$ for some (equivalently, all) $\pi \in \operatorname{Kak}(k, u, v, w)$. For each $m \in \mathbb{N}$, let $G_{m}$ denote the set of functions $g: S \rightarrow 2^{m}$ such that $g(0, s) \neq g(i, s)$ for all $(i, s) \in S$ with $i \in \mathbb{Z}^{+}$. For each $g \in G_{m}$, let $\mathcal{A}_{g, m}$ denote the set of $\pi \in \mathcal{A}$ such that $g(i, s)=f^{i} \circ \pi(s) \mid m$ for all $(i, s) \in S$. As $\mathcal{A} \subseteq \bigcup_{m \in \mathbb{N}} \bigcup_{g \in G_{m}} \mathcal{A}_{g, m}$, there exist $m \in \mathbb{N}$ and $g \in G_{m}$ such that $\mathcal{A}_{g, m} \notin \mathcal{I}_{\sigma_{d, n}}$. Set $\mathcal{A}^{\prime}=\mathcal{A}_{g, m}$.

Observe now that if $\pi_{0}, \pi_{1} \in \mathcal{A}^{\prime}$ and $\pi_{0}\left(s_{n}\right)<_{f} \pi_{1}\left(s_{n}\right)$, then the fact that $g \in G_{m}$ ensures that $f^{n} \circ \pi_{0}\left(d 1^{n}\right)<_{f} \pi_{1}\left(s_{n}\right)$. Let $\pi$ denote the Kakutani embedding of $\sigma_{d, n+1}$ into $f$ given by $\pi(s i)=\pi_{i}(s)$. As there is an extension $\left(k^{\prime}, u^{\prime}, v^{\prime}, w^{\prime}\right)$ of $(k, u, v, w)$ with $\pi \in \operatorname{Kak}\left(k^{\prime}, u^{\prime}, v^{\prime}, w^{\prime}\right)$, the definition of $B$ ensures that there exist $i<2$ and $s \in(d+1) \times 2^{n}$ such that $\pi_{i}(s) \in B$, which contradicts the fact that $\pi_{i} \in \mathcal{A}$. It therefore follows that the set $A=\left\{\pi\left(s_{n}\right): \pi \in \mathcal{A}^{\prime}\right\}$ is an antichain. As the property of being an antichain is co-analytic on analytic, the first reflection theorem (see, for example, Theorem 35.10 of [11]) implies that $A$ is contained in a Borel antichain, thus $\mathcal{A}^{\prime} \in \mathcal{I}_{\sigma_{d, n}}$, the desired contradiction.

If $\mathcal{I}_{d, 0}$ does not trivialize, then Lemma 3.7 ensures that there is a sequence of reasonable $(d, n)$-configurations $\left(k_{n}, u_{n}, v_{n}, w_{n}\right)$, each of which is extended by the next. Define a continuous function $\pi_{\infty}:(d+1) \times 2^{\mathbb{N}} \rightarrow \mathbb{N}^{\mathbb{N}}$ by

$$
\pi_{\infty}(x)=\lim _{n \rightarrow \infty} u_{n}(x \mid(n+1)) .
$$

Lemma 3.8. Suppose that $c<d$ and $x \in 2^{\mathbb{N}}$. Then $\pi_{\infty}(d x)=f^{v_{0}(c)} \circ \pi_{\infty}(c x)$.

Proof. By the continuity of $f$, it is enough to show that if $U$ is an open neighborhood of $\pi_{\infty}(c x)$ and $V$ is an open neighborhood of $\pi_{\infty}(d x)$, then there exist $y \in U$ and $z \in V$ such that $z=f^{v_{0}(c)}(y)$. Towards this end, fix $n \in \mathbb{N}$ sufficiently large that $\mathcal{N}_{u_{n}(c(x \mid n))} \subseteq U$ and $\mathcal{N}_{u_{n}(d(x \mid n))} \subseteq V$, fix $\pi \in \operatorname{Kak}\left(k_{n}, u_{n}, v_{n}, w_{n}\right)$, and observe that $y=\pi(c(x \mid n))$ and $z=\pi(d(x \mid n))$ are as desired.

Lemma 3.9. Suppose that $m \in \mathbb{N}$ and $x \in 2^{\mathbb{N}}$. Then $\pi_{\infty}\left(s_{m} 1 x\right)=f^{w_{m+1}(m)} \circ \pi_{\infty}\left(d 1^{m} 0 x\right)$.

Proof. By the continuity of $f$, it is enough to show that if $U$ is an open neighborhood of $\pi_{\infty}\left(d 1^{m} 0 x\right)$ and $V$ is an open neighborhood of $\pi_{\infty}\left(s_{m} 1 x\right)$, then there exist $y \in U$ and $z \in V$ such that $z=f^{w_{m+1}(m)}(y)$. Towards this end, fix $n \in \mathbb{N}$ sufficiently large that $\mathcal{N}_{u_{m+1+n}\left(d 1^{m} 0(x \mid n)\right)} \subseteq U$ and $\mathcal{N}_{u_{m+1+n}\left(s_{m} 1(x \mid n)\right)} \subseteq V$, fix $\pi \in$ $\operatorname{Kak}\left(k_{m+1+n}, u_{m+1+n}, v_{m+1+n}, w_{m+1+n}\right)$, and observe that $y=\pi\left(d 1^{m} 0(x \mid n)\right)$ and $z=\pi\left(s_{m} 1(x \mid n)\right)$ are as desired. 
Lemma 3.10. Suppose that $x, y \in(d+1) \times 2^{\mathbb{N}}, x(n) \neq y(n)$, and $i, j \leq n$. Then $f^{i} \circ \pi_{\infty}(x) \neq f^{j} \circ \pi_{\infty}(y)$.

Proof. The fact that $x(n) \neq y(n)$ ensures that $f^{i}\left(\mathcal{N}_{u_{n}(x \mid(n+1))}\right) \cap f^{j}\left(\mathcal{N}_{u_{n}(y \mid(n+1))}\right)=\emptyset$, thus $f^{i} \circ \pi_{\infty}(x) \neq f^{j} \circ \pi_{\infty}(y)$.

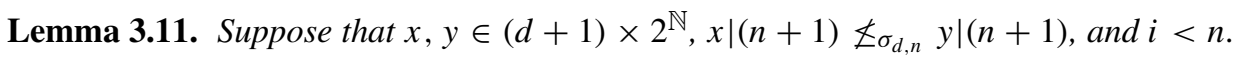
Then $f^{i} \circ \pi_{\infty}(x) \neq \pi_{\infty}(y)$.

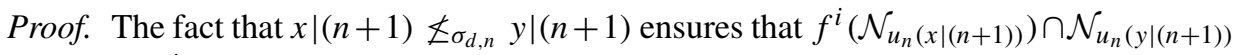
$=\emptyset$, thus $f^{i} \circ \pi_{\infty}(x) \neq \pi_{\infty}(y)$.

Lemmas 3.8 and 3.9 imply that if $x \leq_{\sigma_{d}} y$, then $\pi_{\infty}(x) \leq_{f} \pi_{\infty}(y)$. Lemma 3.10 implies that $\pi_{\infty}$ is injective and if $(x, y) \notin E_{0}$, then $\left(\pi_{\infty}(x), \pi_{\infty}(y)\right) \notin E_{t}(f)$. Lemma 3.11 implies that if $x{\mathbb{E}_{d}} y$ and $x E_{0} y$, then $\pi_{\infty}(x) \mathbb{Z}_{f} \pi_{\infty}(y)$. It follows that $\pi_{\infty}$ is the desired Kakutani embedding.

Theorem 3.12. Suppose that $X$ is a Polish space and $f: X \rightarrow X$ is a Borel function. Then exactly one of the following holds:

(1) The function $f$ is antichainable.

(2) There is a continuous Kakutani embedding of $\sigma_{1}$ into $f$.

Proof. In light of Theorem 3.6, it is enough to show that if $\mathcal{I}_{\sigma_{1,0}}$ trivializes, then $f$ is antichainable. Towards this end, suppose that there are countably many Borel antichains whose union $A$ intersects the range of every Kakutani embedding of $\sigma_{1,0}$ into $f$, and observe that the complement of $A$ is necessarily an antichain (since otherwise there is a Kakutani embedding of $\sigma_{1,0}$ into $f$ whose range is disjoint from $A$ ), thus $f$ is antichainable.

We can now tie together several different properties of Borel functions:

Theorem 3.13. Suppose that $X$ is a Polish space and $f: X \rightarrow X$ is an aperiodic Borel function. Then the following are equivalent:

(1) The function $f$ is antichainable.

(2) There is no continuous Kakutani embedding of $\sigma$ into $f$.

(3) There is a decreasing sequence $\left\langle B_{n}\right\rangle_{n \in \mathbb{N}}$ of complete, stable Borel sets with empty intersection.

Moreover, if $f$ is countable-to-one, then these are equivalent to:

(4) There is a Borel embedding of $f$ into $s_{(\mathbb{N})}$.

(5) There is a Kakutani embedding of $f$ into $s_{(\mathbb{N})}$.

Proof. To see (1) $\Leftrightarrow$ (2), simply note that $\sigma$ and $\sigma_{1}$ are continuously Kakutani bi-embeddable, and appeal to Theorem 3.12 To see (1) $\Rightarrow(3)$, fix Borel antichains $A_{n}$ whose union is $X$, and note that the sets

$$
B_{n}=X \backslash \bigcup_{m<n} \bigcup_{i \in \mathbb{N}} f^{-i}\left(A_{m}\right)
$$


are as desired. To see (3) $\Rightarrow(1)$, suppose that $\left\langle B_{n}\right\rangle_{n \in \mathbb{N}}$ is a decreasing sequence of complete, stable Borel sets with empty intersection, and observe that the sets

$$
A_{m n}=f^{-(m+1)}\left(B_{n}\right) \backslash \bigcup_{l \leq m} f^{-l}\left(B_{n}\right),
$$

for $m, n \in \mathbb{N}$, are Borel antichains whose union is $X$.

Suppose now that $f$ is countable-to-one. Obviously (4) $\Rightarrow(5)$, and (5) $\Rightarrow$ (1) is a consequence of the fact that antichainability is closed under Kakutani embeddability. To see (1) $\Rightarrow$ (4), fix a partition of $X$ into Borel antichains $A_{n}$, and let $\phi(x)$ denote the unique $n \in \mathbb{N}$ such that $x \in A_{n}$. An $(f$-)generator is a function $\psi: X \rightarrow Y$ such that for all distinct $x_{1}, x_{2} \in X$, there exists $n \in \mathbb{N}$ with $\psi \circ f^{n}\left(x_{1}\right) \neq \psi \circ f^{n}\left(x_{2}\right)$. Theorem 7.6 of [12] ensures that there is a Borel generator $\psi: X \rightarrow \mathbb{N}$. Fix a bijection $\langle\cdot, \cdot\rangle$ from $\mathbb{N} \times \mathbb{N}$ to $\mathbb{N}$, and define $\pi: X \rightarrow(\mathbb{N})^{\mathbb{N}}$ by $[\pi(x)](n)=\left\langle\phi \circ f^{n}(x), \psi \circ f^{n}(x)\right\rangle$. Then $\pi$ is a Borel embedding of $f$ into $s_{(\mathbb{N})}$.

We close this section with one more dichotomy theorem:

Theorem 3.14. Suppose that $X$ is a Polish space and $f: X \rightarrow X$ is a Borel function. Then exactly one of the following holds:

(1) The function $f$ can be decomposed into antichainable and essentially injective parts.

(2) There is a continuous Kakutani embedding of $\sigma_{2}$ into $f$.

Proof. In light of Theorem 3.6 it is enough to show that if $\mathcal{I}_{\sigma_{2,0}}$ trivializes, then $f$ can be decomposed into antichainable and essentially injective parts. Towards this end, suppose that there are Borel antichains $B_{n} \subseteq X$ whose union intersects the range of every Kakutani embedding of $\sigma_{2,0}$ into $f$, set $B=\bigcup_{n \in \mathbb{N}} \bigcup_{i \in \mathbb{N}} f^{-i}\left(B_{n}\right)$, and observe that the set $X \backslash B$ is necessarily linear (since otherwise there is a Kakutani embedding of $\sigma_{2,0}$ into $f$ whose range is disjoint from $B$ ), thus $f$ can be decomposed into antichainable and essentially injective parts.

Remark 3.15. In the statement of Theorem 3.14 the map $\sigma_{2}$ can be replaced with $\sigma_{d}$ for any $d \geq 2$. This follows from Theorems 3.6 and 3.14 and the observation that the $\sigma$-ideal $\mathcal{I}_{\sigma_{d}}$ corresponding to the function $f=\sigma_{2}$ does not trivialize.

\section{Maximality of many-to-one odometers}

In this section, we characterize the Kakutani equivalence class of $\sigma_{2}$.

Theorem 4.1. Suppose that $X$ is a Polish space and $f: X \rightarrow X$ is an aperiodic, countable-to-one Borel function. Then $f \sqsubseteq_{K} \sigma_{2}$.

Proof. We say that a set $C \subseteq X$ is $\left(f\right.$-) convex if it is contained in a single $E_{t}(f)$-class and $x \leq_{f} \quad y$ for all $x \in C$ and $y \in C \backslash f^{-1}(C)$. We say that a Kakutani embedding $\pi: C \rightarrow 3 \times 2^{n}$ of $f \mid C$ into $\sigma_{2, n}$ is extendable if for all $x \in C$, there exists $s \in D_{2, n} \backslash R_{2, n}$ such that $\pi(x)$ is the $\leq_{\sigma_{2, n}}$-minimal iterate of $s$ in $\pi(C)$. An m-extension problem is a quintuple $\left(C, C_{0}, C_{1}, \pi_{0}, \pi_{1}\right)$ such that: 
(a) The sets $C, C_{0}$, and $C_{1}$ are convex, finite, and non-empty, and $C$ is the disjoint union of $C_{0}$ and $C_{1}$.

(b) The functions $\pi_{0}$ and $\pi_{1}$ are extendable Kakutani embeddings of $f \mid C_{0}$ and $f \mid C_{1}$ into $\sigma_{2, m}$.

For each $x \in C$, let $i(x)$ denote the unique $i \in\{0,1\}$ such that $x \in C_{i}$. An $n$-solution is a pair $\left(t_{0}, t_{1}\right)$, where $t_{0}, t_{1} \in 2^{n-m}$, such that the map $x \mapsto \pi_{i(x)}(x) t_{i(x)}$ is an extendable Kakutani embedding of $f \mid C$ into $\sigma_{2, n}$.

Lemma 4.2. Suppose that $m \in \mathbb{N}$. Then there exists $n \geq m$ such that every $m$-extension problem has an $n$-solution.

Proof. As there are essentially only finitely many $m$-extension problems, we need only show that for every $m$-extension problem $\left(C, C_{0}, C_{1}, \pi_{0}, \pi_{1}\right)$, there exists $n \in \mathbb{N}$ such that there is an $n$-solution. By reversing the roles of $C_{0}$ and $C_{1}$ if necessary, we can assume that $C_{0} \cap f^{-1}\left(C_{1}\right) \neq \emptyset$. By the extendability of $\pi_{1}$, there exists $s \in D_{2, m} \backslash R_{2, m}$ such that $\pi_{1} \circ f(x)$ is the $\leq_{\sigma_{2, m}}$-minimal iterate of $s$ in $\pi_{1}\left(C_{1}\right)$. Fix $l \geq m$ and $t \in 2^{l-m}$ such that $s_{l}=s t$, as well as distinct sequences $u, v \in D_{2, l} \backslash R_{2, l}$, noting that $u 0, v 0 \leq \sigma_{2, l+1}$ st 1 . Fix $k>l$ and $w \in 2^{k-(l+1)}$ such that $s_{k}=u 0 w$, set $n=k+1$, and observe that

$$
\pi_{0}(x) 0^{k-m} 0 \leq_{\sigma_{2, n}} d 1^{k} 0 \leq_{\sigma_{2, n}} u 0 w 1 \leq_{\sigma_{2, n}} \text { st } 1 w 1 \leq_{\sigma_{2, n}}\left(\pi_{1} \circ f(x)\right) t 1 w 1,
$$

thus $\left(0^{n-m}, t 1 w 1\right)$ is the desired $n$-solution.

We say that a subequivalence relation $F$ of $E_{t}(f)$ is $(f$-)convex if all of its equivalence classes are convex. Note that every such subequivalence relation is entirely determined by $f$ and the set $\{x \in X: x F f(x)\}$. The trivial equivalence relation on $X$ is given by $\Delta(X)=\{(x, x): x \in X\}$.

Lemma 4.3. There is an increasing sequence of convex finite Borel equivalence relations $F_{n}$ on $X$ such that:

(1) The equivalence relation $F_{0}$ is trivial.

(2) Every $F_{n+1}$-class is the (not necessarily disjoint) union of two $F_{n}$-classes.

(3) The union of the equivalence relations $F_{n}$ is $E_{t}(f)$.

Proof. By Corollary 8.2 of [3], there is an increasing sequence of finite Borel equivalence relations $F_{k}^{\prime}$ on $X$ such that $F_{0}^{\prime}=\Delta(X)$ and $E_{t}(f)=\bigcup_{k \in \mathbb{N}} F_{k}^{\prime}$. Let $F_{k}^{\prime \prime}$ denote the convex equivalence relation given by $x F_{k}^{\prime \prime} f(x) \Leftrightarrow x F_{k}^{\prime} f(x)$. It is clear that $F_{0}^{\prime \prime}=\Delta(X)$, the sequence $\left\langle F_{k}^{\prime \prime}\right\rangle_{k \in \mathbb{N}}$ is increasing, and $E_{t}(f)=\bigcup_{k \in \mathbb{N}} F_{k}^{\prime \prime}$.

We will now recursively construct convex finite Borel equivalence relations $F_{n}$ on $X$ with the additional property that

$$
\forall k \in \mathbb{N} \forall x \in X\left([x]_{F_{k}^{\prime \prime}} \subseteq[x]_{F_{n}} \text { or }[x]_{F_{n}} \subseteq[x]_{F_{k}^{\prime \prime}}\right) .
$$

We begin by setting $F_{0}=\Delta(X)$. Fix a Borel linear ordering $\leq$ of $X$. Given $F_{n}$, let $k_{n}(x)$ denote the least natural number $k$ such that $[x]_{F_{k}^{\prime \prime}} \nsubseteq[x]_{F_{n}}$. It is clear that $x F_{n} y \Rightarrow$ 
$k_{n}(x)=k_{n}(y)$, so we can think of $k_{n}$ as a function which associates a natural number to each $F_{n}$-class within the $F_{k_{n}(x)}^{\prime \prime}$-class of $x$. We now restrict our attention to the set

$$
A_{n}=\left\{x \in X: \forall y \in[x]_{F_{k_{n}(x)}^{\prime \prime}}\left(k_{n}(x) \leq k_{n}(y)\right)\right\}
$$

of points whose $F_{n}$-classes are assigned minimal index within their $F_{k_{n}(x)}^{\prime \prime}$-classes. Note that if $x \in A_{n}$, then $k_{n} \mid[x]_{F_{k_{n}(x)}^{\prime \prime}}$ is constant, thus $[x]_{F_{k_{n}(x)}^{\prime \prime}} \subseteq A_{n}$. Set

$$
B_{n}=\left\{x \in A_{n}:(x, f(x)) \in F_{k_{n}(x)}^{\prime \prime} \backslash F_{n}\right\} .
$$

We would like to include the graph of $f \mid B_{n}$ in $F_{n+1}$, but this could potentially lead to a violation of condition (2). Instead, we restrict our attention to the set

$$
C_{n}=\left\{x \in B_{n}: \forall y \in B_{n} \cap[x]_{F_{k_{n}(x)}^{\prime \prime}}(x \leq y)\right\} .
$$

Define $F_{n+1}$ on $X$ by $x F_{n+1} f(x) \Leftrightarrow\left(x F_{n} f(x)\right.$ or $\left.x \in C_{n}\right)$.

It remains to check that the equivalence relation $F=\bigcup_{n \in \mathbb{N}} F_{n}$ is $E_{t}(f)$. Suppose, towards a contradiction, that this is not the case, fix $k \in \mathbb{N}$ least such that $F_{k}^{\prime \prime} \nsubseteq F$, and fix $x \in X$ such that $[x]_{F_{k}^{\prime \prime}} \nsubseteq[x]_{F}$. Then (†) ensures that $[x]_{F_{k}^{\prime \prime}}$ is $F$-invariant. Fix $n \in \mathbb{N}$ sufficiently large that $[y]_{F_{n}}=[y]_{F}$ for all $y \in[x]_{F_{k}^{\prime \prime}}$, and let $y$ denote the $\leq$-minimal element of $[x]_{F_{k}^{\prime \prime}}$ such that $(y, f(y)) \notin F$. Then $y \in C_{n}$, thus $y F_{n+1} f(y)$, the desired contradiction.

Set $k_{0}=0$. Given $k_{n} \in \mathbb{N}$, put $l_{n}=3 n \cdot 2^{k_{n}}$ and fix $k_{n+1} \geq k_{n}+l_{n}$ sufficiently large that every $\left(k_{n}+l_{n}\right)$-extension problem has a $k_{n+1}$-solution.

We can clearly assume that $X=2^{\mathbb{N}}$. We will recursively construct Borel functions $\pi_{n}: 2^{\mathbb{N}} \rightarrow 3 \times 2^{k_{n}}$ such that $\pi_{n} \mid[x]_{F_{n}}$ is an extendable Kakutani embedding of $f \mid[x]_{F_{n}}$ into $\sigma_{2, k_{n}}$, for all $x \in 2^{\mathbb{N}}$. Define $\pi_{0}: 2^{\mathbb{N}} \rightarrow 3$ by $\pi_{0}(x)=2$.

Suppose now that we have already defined $\pi_{n}: X \rightarrow 3 \times 2^{k_{n}}$. For all $x \in 2^{\mathbb{N}}$, define $\phi_{n}(x): 3 \times 2^{k_{n}} \rightarrow 2^{n}$ by

$$
\left[\phi_{n}(x)\right](s)= \begin{cases}\left(\pi_{n} \mid[x]_{F_{n}}\right)^{-1}(s) \mid n & \text { if } s \in \pi_{n}\left([x]_{F_{n}}\right), \\ 0^{n} & \text { otherwise. }\end{cases}
$$

As $l_{n}=3 n \cdot 2^{k_{n}}$, we can think of $\phi_{n}$ as a map from $2^{\mathbb{N}}$ into $2^{l_{n}}$. As $x F_{n} y \Rightarrow \phi_{n}(x)=$ $\phi_{n}(y)$, it follows that the map $y \mapsto \pi_{n}(y) \phi_{n}(y)$ is an extendable Kakutani embedding of $f \mid[x]_{F_{n}}$ into $\sigma_{2, k_{n}+l_{n}}$.

If $[x]_{F_{n}}=[x]_{F_{n+1}}$ and we set $\psi_{n}(x)=0^{k_{n+1}-\left(k_{n}+l_{n}\right)}$, then the map $y \mapsto$ $\pi_{n}(y) \phi_{n}(y) \psi_{n}(y)$ is an extendable Kakutani embedding of $f \mid[x]_{F_{n+1}}$ into $\sigma_{2, k_{n+1}}$. Otherwise, there exists $\left(x_{0}, x_{1}\right) \notin F_{n}$ such that $[x]_{F_{n+1}}=\left[x_{0}\right]_{F_{n}} \cup\left[x_{1}\right]_{F_{n}}$, in which case there are sequences $t_{i} \in 2^{k_{n+1}-\left(k_{n}+l_{n}\right)}$ such that the map $y \mapsto \pi_{n}(y) \phi_{n}(y) \psi_{n}(y)$, where $\psi_{n}(y)=t_{i}$ for $y \in\left[x_{i}\right]_{F_{n}}$, is an extendable Kakutani embedding of $f \mid[x]_{F_{n+1}}$ into $\sigma_{2, k_{n+1}}$. It follows that there is an $F_{n}$-invariant Borel function $\psi_{n}: 2^{\mathbb{N}} \rightarrow 2^{k_{n+1}-\left(k_{n}+l_{n}\right)}$ such that the map $\pi_{n+1}(x)=\pi_{n}(x) \phi_{n}(x) \psi_{n}(x)$ is as desired.

Define now $\pi: 2^{\mathbb{N}} \rightarrow 3 \times 2^{\mathbb{N}}$ by $\pi(x)=\lim _{n \rightarrow \infty} \pi_{n}(x)$. We will show that $\pi$ is the desired Kakutani embedding of $f$ into $\sigma_{2}$. 
Lemma 4.4. Suppose that $x<_{f} y$. Then $\pi(x)<_{\sigma_{2}} \pi(y)$.

Proof. Fix $n \in \mathbb{N}$ such that $x F_{n} y$. Then $\pi_{n}(x)<_{\sigma_{2, k_{n}}} \pi_{n}(y)$, and since $[\pi(x)](i)=$ $[\pi(y)](i)$ for all $i>k_{n}$, it follows that $\pi(x)<_{\sigma_{2}} \pi(y)$.

It remains to check that $\pi(x) \leq_{\sigma_{2}} \pi(y) \Rightarrow x \leq_{f} y$. We can clearly assume that $x \neq y$. Fix $n \in \mathbb{N}$ sufficiently large that at least one of $x \mid n$ or $y \mid n$ is not $0^{n}$ and $\phi_{m}(x) \psi_{m}(x)=$ $\phi_{m}(y) \psi_{m}(y)$, for all $m \geq n$. We will assume that $x \mid n \neq 0^{n}$, as the other case is handled similarly. As $x \mid n=\left[\phi_{n}(y)\right]\left(\pi_{n}(x)\right) \neq 0^{n}$, there exists $z \in[y]_{F_{n}}$ such that $\pi_{n}(x)=$ $\pi_{n}(z)$. It follows that if $m \geq n$, then $\pi_{m}(x)=\pi_{m}(z)$, so $x \mid m=\left[\phi_{m}(y)\right]\left(\pi_{m}(x)\right)=$ $\left[\phi_{m}(y)\right]\left(\pi_{m}(z)\right)=z \mid m$, thus $x=z$, which implies that $x F_{n} y$. As $\pi(x) \leq_{\sigma_{2}} \pi(y)$, it follows that $\pi_{n}(x) \leq_{\sigma_{2, k_{n}}} \pi_{n}(y)$, thus $x \leq_{f} y$.

As a corollary, we obtain the following:

Theorem 4.5. All aperiodic, essentially countable-to-one Borel functions on Polish spaces which cannot be decomposed into antichainable and essentially injective parts are Kakutani bi-reducible. Moreover, the class of such functions is closed under Kakutani equivalence.

Proof. Suppose first that $X$ and $Y$ are Polish spaces and $f: X \rightarrow X$ and $g: Y \rightarrow Y$ are aperiodic, essentially countable-to-one Borel functions which cannot be decomposed into antichainable and essentially injective parts. Fix a complete, recurrent Borel set $A \subseteq X$ such that $f_{A}$ is countable-to-one. A straightforward induction shows that $\bigcup_{i \leq n} f^{i}(A)$ is a recurrent Borel set whose induced transformation is countable-to-one, thus $[A]_{f}$ is a stable Borel set whose induced transformation is countable-to-one. By replacing $A$ with its forward saturation, we can therefore assume that $A$ is stable. Theorems 3.14 and 4.1 then imply that $f_{A} \sqsubseteq_{K} \sigma_{2} \sqsubseteq_{K} g$, thus symmetry ensures that $f \simeq_{K} g$.

Suppose now that $f$ and $g$ are Kakutani equivalent aperiodic Borel functions on Polish spaces. If $g$ can be decomposed into antichainable and essentially injective parts, then the fact that antichains and linear sets can be pulled back through Kakutani embeddings ensures that $f$ also admits such a decomposition.

It is a simple task to establish that the restriction of $\sigma_{2}$ to the non-eventually constant sequences is Kakutani equivalent to $\sigma_{2}$. As the former function is total, we can safely think of $\sigma_{2}$ as a total function.

\section{The shift on increasing sequences of natural numbers}

In this section, we study Kakutani embeddability and equivalence of $s_{[\mathbb{N}]}$. While there is a natural strategy for embedding $s_{[\mathbb{N}]}$ directly, it will simplify matters to work instead with the product of the unilateral shift $s$ on $2^{\mathbb{N}}$ with the successor on $\mathbb{N}$, i.e., the function $\sigma_{\perp}: 2^{\mathbb{N}} \times \mathbb{N} \rightarrow 2^{\mathbb{N}} \times \mathbb{N}$ given by $\sigma_{\perp}(x, n)=(s(x), n+1)$.

Proposition 5.1. There is a continuous embedding of $\sigma_{\perp}$ into $s_{[\mathbb{N}]}$. 
Proof. Define $\pi: 2^{\mathbb{N}} \times \mathbb{N} \rightarrow[\mathbb{N}]^{\mathbb{N}}$ by $[\pi(x, m)](n)=x(n)+2(m+n)$. It is clear that $\pi$ is a continuous injection, and if $n \in \mathbb{N}$ and $(x, m) \in 2^{\mathbb{N}} \times \mathbb{N}$, then

$$
\left[s_{[\mathbb{N}]} \circ \pi(x, m)\right](n)=x(n+1)+2(m+n+1)=\left[\pi \circ \sigma_{\perp}(x, m)\right](n),
$$

thus $\pi$ is an embedding of $\sigma_{\perp}$ into $s_{[\mathbb{N}]}$.

Proposition 5.2. There is a continuous Kakutani embedding of $s_{[\mathbb{N}]}$ into $\sigma_{\perp}$.

Proof. It is sufficient to show that there is a continuous isomorphism of $s_{[\mathbb{N}]}$ and $\left(\sigma_{\perp}\right)_{B}$, where $B=\left\{(x, n) \in 2^{\mathbb{N}} \times \mathbb{N}: x(0)=1\right.$ and $\left.\forall n \in \mathbb{N} \exists m \geq n(x(m)=1)\right\}$. Towards this end, define $\pi:[\mathbb{N}]^{\mathbb{N}} \rightarrow 2^{\mathbb{N}} \times \mathbb{N}$ by $\pi(x)=\left(\bigoplus_{n \in \mathbb{N}} 10^{\bar{x}(n+1)-x(n)-1}, x(0)\right)$. It is clear that $\pi$ is a continuous injection, $B=\pi\left([\mathbb{N}]^{\mathbb{N}}\right)$, and if $x \in[\mathbb{N}]^{\mathbb{N}}$, then

$$
\begin{aligned}
\left(\sigma_{\perp}\right)_{B} \circ \pi(x) & =\left(\sigma_{\perp}\right)_{B}\left(\bigoplus_{n \in \mathbb{N}} 10^{x(n+1)-x(n)-1}, x(0)\right)=\left(\bigoplus_{n \in \mathbb{N}} 10^{x(n+2)-x(n+1)-1}, x(1)\right) \\
& =\pi \circ S_{[\mathbb{N}]}(x),
\end{aligned}
$$

thus $\pi$ is an isomorphism of $s_{[\mathbb{N}]}$ and $\left(\sigma_{\perp}\right)_{B}$.

A homomorphism from $f: X \rightarrow X$ to $g: Y \rightarrow Y$ is a (not necessarily injective) function $\pi: X \rightarrow Y$ such that $\pi \circ f=g \circ \pi$.

Proposition 5.3. There is no Borel homomorphism from $s_{[\mathbb{N}]}$ to $\sigma_{\perp}$.

Proof. Recall that a directed graph on $X$ is an irreflexive set $\mathcal{G} \subseteq X \times X$. A coloring of $\mathcal{G}$ is a function $c: X \rightarrow Y$ such that $c(x) \neq c(y)$ for all $(x, y) \in \mathcal{G}$. When $X$ is a Polish space, the Borel chromatic number of $\mathcal{G}$, or $\chi_{B}(\mathcal{G})$, is the least cardinal of the form $|Y|$, where $Y$ is a Polish space and $c: X \rightarrow Y$ is a Borel coloring of $\mathcal{G}$.

It is clear that $\chi_{B}\left(\operatorname{graph}\left(\sigma_{\perp}\right)\right)=2$ and, as noted in Example 3.2 of [12], the GalvinPrikry theorem [7] implies that $\chi_{B}\left(\operatorname{graph}\left(s_{[\mathbb{N}]}\right)\right)=\aleph_{0}$. As colorings can be pulled back through homomorphisms, the proposition follows.

Remark 5.4. While the proof of Proposition 5.2 easily implies that there is a complete, recurrent Borel set $B \subseteq 2^{\mathbb{N}} \times \mathbb{N}$ such that $s_{[\mathbb{N}]} \cong_{B}\left(\sigma_{\perp}\right)_{B}$, the Galvin-Prikry theorem [7] ensures that if $B \subseteq[\mathbb{N}]^{\mathbb{N}}$ is a complete, recurrent Borel set, then $s_{[\mathbb{N}]} \sqsubseteq{ }_{B} s_{B}$, thus $\sigma_{\perp} \varlimsup_{B}\left(s_{[\mathbb{N}]}\right)_{B}$, by Proposition 5.3

We are now ready for the main result of this section:

Theorem 5.5. Suppose that $X$ is a Polish space and $f: X \rightarrow X$ is Borel. Then exactly one of the following holds:

(1) The function $f$ is essentially injective.

(2) There is a continuous Kakutani embedding of $\sigma_{\perp}$ into $f$. 
Proof. To see that (1) and (2) are mutually exclusive, suppose that $f$ is essentially injective and $\pi$ is a Kakutani embedding of $\sigma_{\perp}$ into $f$. Fix a complete, linear Borel set $B \subseteq X$, and observe that the Borel set $C=[B]_{f}^{\rightarrow}$ is linear. Fix $k, n \in \mathbb{N}$ and $t \in 2^{k}$ such that the set $A=\pi^{-1}(C)$ is comeager in $\mathcal{N}_{t} \times\{n\}$, fix $x \in 2^{\mathbb{N}}$ such that $(t 0 x, n),(t 1 x, n) \in A$, and note that $(x, k+n+1)=\sigma_{\perp}^{k+1}(t 0 x, n)=\sigma_{\perp}^{k+1}(t 1 x, n)$, which contradicts the fact that $A$ is linear and stable.

It remains to show $\neg(1) \Rightarrow(2)$. By standard change of topology results, we can assume that $f$ is continuous. We also assume that $X=\mathbb{N}^{\mathbb{N}}$, as the general case is handled similarly. Let $s_{n}$ denote the shift on $2^{\leq n}$.

An $n$-configuration is a triple $(k, u, v)$, where $k \in \mathbb{N}, u: 2^{n} \rightarrow \mathbb{N}^{k}$, and $v: n \times 2 \rightarrow \mathbb{N}$, with the property that

$$
f^{j+\sum_{i<l} v(i, 0)}\left(\mathcal{N}_{u\left(0^{l} s\right)}\right) \cap f^{\sum_{i<m} v(i, 0)}\left(\mathcal{N}_{u\left(0^{m} t\right)}\right)=\emptyset
$$

for all $j, l, m \leq n, s \in 2^{n-l}$, and $t \in 2^{n-m}$ such that $s \mathbb{L}_{s_{n}} t$.

An extension of $(k, u, v)$ is an $(n+1)$-configuration $\left(k^{\prime}, u^{\prime}, v^{\prime}\right)$ such that $u(s) \sqsubseteq u^{\prime}(s i)$ and $v=v^{\prime} \mid(n \times 2)$, for all $i<2$ and $s \in 2^{n}$.

$\mathrm{A}(k, u, v)$-embedding is a Kakutani embedding $\pi$ of $s_{n}$ into $f$ such that $\pi(s) \in \mathcal{N}_{u(s)}$ and $\pi(t)=f^{v(n-m-1, j)} \circ \pi(j t)$, for all $j<2, m<n, s \in 2^{n}$, and $t \in 2^{m}$.

We say that $(k, u, v)$ is reasonable if there is no linear Borel set $B \subseteq \mathbb{N}^{\mathbb{N}}$ whose saturation contains the range of every $(k, u, v)$-embedding.

Lemma 5.6. Every reasonable n-configuration has a reasonable extension.

Proof. Suppose, towards a contradiction, that $(k, u, v)$ is a reasonable $n$-configuration, and there is a linear Borel set $D \subseteq \mathbb{N}^{\mathbb{N}}$ whose saturation contains the range of every $\left(k^{\prime}, u^{\prime}, v^{\prime}\right)$-embedding, for every extension $\left(k^{\prime}, u^{\prime}, v^{\prime}\right)$ of $(k, u, v)$.

Sublemma 5.7. There are $(k, u, v)$-embeddings $\pi_{0}$ and $\pi_{1}$, whose ranges are disjoint from $[D]_{f}$ and contained in the same $E_{t}(f)$-class, such that $\pi_{0}(\emptyset) \perp_{f} \pi_{1}(\emptyset)$.

Proof. Let $A$ denote the set of points of the form $\pi(\emptyset)$, where $\pi$ is a $(k, u, v)$-embedding whose range is disjoint from $[D]_{f}$. Suppose, towards a contradiction, that $A$ is linear. As linearity is co-analytic on analytic, the first reflection theorem implies that there is a linear Borel set containing $A$, which contradicts the reasonability of $(k, u, v)$.

Fix $\pi_{0}$ and $\pi_{1}$ as in the conclusion of Sublemma 5.7. fix a Kakutani embedding $\pi$ of $s_{n+1}$ into $f$ such that $\pi(t i)=\pi_{i}(t)$, for all $i<2$ and $t \in 2^{\leq n}$, and observe that there is an extension $\left(k^{\prime}, u^{\prime}, v^{\prime}\right)$ of $(k, u, v)$ such that $\pi$ is a $\left(k^{\prime}, u^{\prime}, v^{\prime}\right)$-embedding. Then $\pi\left(2^{n+1}\right) \subseteq$ $[D]_{f}$, the desired contradiction.

Lemma 5.6 ensures that if $f$ is not essentially injective, then there is a sequence of reasonable $n$-configurations $\left(k_{n}, u_{n}, v_{n}\right)$, each of which is extended by the next. Define a continuous function $\pi_{\infty}: 2^{\mathbb{N}} \times \mathbb{N} \rightarrow \mathbb{N}^{\mathbb{N}}$ by

$$
\pi_{\infty}(x, 0)=\lim _{n \rightarrow \infty} u_{n}(x \mid n) \quad \text { and } \quad \pi_{\infty}(x, m)=f^{\sum_{i<m} v_{m}(i, 0)} \circ \pi_{\infty}\left(0^{m} x, 0\right) .
$$


Lemma 5.8. Suppose that $j, m \in \mathbb{N}$ and $x \in \mathbb{N}^{\mathbb{N}}$. Then $\pi_{\infty}(x, m+1)=f^{v_{m+1}(m, j)}$ 。 $\pi_{\infty}(j x, m)$.

Proof. By the continuity of $f$, it is enough to show that if $U$ is an open neighborhood of $\pi_{\infty}(j x, m)$ and $V$ is an open neighborhood of $\pi_{\infty}(x, m+1)$, then there exist $y \in U$ and $z \in V$ such that $z=f^{v_{m+1}(m, j)}(y)$. Towards this end, fix $n>m$ such that

$$
f^{\sum_{i<m} v_{m}(i, 0)}\left(\mathcal{N}_{u_{n}\left(\left(0^{m} j x\right) \mid n\right)}\right) \subseteq U \quad \text { and } \quad f^{\sum_{i \leq m} v_{m+1}(i, 0)}\left(\mathcal{N}_{u_{n}\left(\left(0^{m+1} x\right) \mid n\right)}\right) \subseteq V,
$$

as well as a $\left(k_{n}, u_{n}, v_{n}\right)$-embedding $\pi$, set $y=\pi((j x) \mid(n-m))$ and $z=\pi(x \mid(n-m-1))$, and observe that $z=f^{v_{n}(m, j)} \circ \pi((j x) \mid(n-m))=f^{v_{m+1}(m, j)}(y)$.

Lemma 5.8 implies that if $(x, l) \leq_{\sigma_{\perp}}(y, m)$, then $\pi_{\infty}(x, l) \leq_{f} \pi_{\infty}(y, m)$. Conversely, suppose that $(x, l) \grave{\leq}_{\sigma_{\perp}}(y, m)$. If $j \in \mathbb{N}$ and $n \geq \max (j, l, m)$ is sufficiently large that $x\left|(n-l) \mathbb{L}_{s_{n}} y\right|(n-m)$, then

$$
f^{j+\sum_{i<l} v_{l}(i, 0)}\left(\mathcal{N}_{u_{n}\left(\left(0^{l} x\right) \mid n\right)}\right) \cap f^{\sum_{i<m} v_{m}(i, 0)}\left(\mathcal{N}_{u_{n}\left(\left(0^{m} y\right) \mid n\right)}\right)=\emptyset,
$$

so $f^{j} \circ \pi_{\infty}(x, l) \neq \pi_{\infty}(y, m)$, thus $\pi_{\infty}$ is the desired Kakutani embedding.

We observe next the corresponding result for the shift:

Theorem 5.9. Suppose that $X$ is a Polish space and $f: X \rightarrow X$ is an aperiodic Borel function. Then exactly one of the following holds:

(1) The function $f$ is essentially injective.

(2) There is a continuous Kakutani embedding of $s_{[\mathbb{N}]}$ into $f$.

Proof. As Propositions 5.1 and 5.2 imply that $\sigma_{\perp}$ and $s_{[\mathbb{N}]}$ are continuously Kakutani biembeddable, the desired result follows from Theorem 5.5

This leads us to a new dichotomy theorem for smoothness:

Theorem 5.10. Suppose that $X$ is a Polish space and $f: X \rightarrow X$ is Borel. Then exactly one of the following holds:

(1) The function $f$ is smooth.

(2) There is a continuous Kakutani embedding of $\sigma$ or $s_{[\mathbb{N}]}$ into $f$.

Proof. To see (1) $\Rightarrow \neg(2)$, observe that if $f$ is smooth, then $f$ is antichainable and essentially injective. As neither $\sigma$ nor $s_{[\mathbb{N}]}$ has both of these properties, it follows that neither $\sigma$ nor $s_{[\mathbb{N}]}$ is Kakutani embeddable into $f$.

To see $\neg(2) \Rightarrow(1)$, we first note the following:

Lemma 5.11. Suppose that $X$ is a Polish space and $f: X \rightarrow X$ is a Borel function which is antichainable and essentially injective. Then $f$ is smooth.

Proof. Fix Borel antichains $B_{n}$ which cover $X$, as well as a complete, linear Borel set $B \subseteq X$, and observe that the set $\bigcup_{n \in \mathbb{N}}\left(B \cap B_{n}\right) \backslash \bigcup_{m<n}\left[B \cap B_{m}\right]_{f}$ is a Borel transversal, thus $f$ is smooth.

The desired result follows from Theorems 3.13 and 5.9 and Lemma 5.11

We have also the following trichotomy: 
Theorem 5.12. Suppose that $X$ is a Polish space and $f: X \rightarrow X$ is an aperiodic, non-smooth Borel function. Then exactly one of the following holds:

(1) The function $f$ is antichainable.

(2) The function $f$ is essentially injective.

(3) There is a continuous Kakutani embedding of $\sigma \oplus s_{[\mathbb{N}]}$ into $f$.

Proof. Lemma 5.11 ensures that (1) and (2) are mutually exclusive, Theorem 3.13 ensures that (1) and (3) are mutually exclusive, and Theorem 5.9 ensures that (2) and (3) are mutually exclusive. Suppose now that both (1) and (2) fail. Theorem 3.13 then ensures that there is an invariant Borel set $B \subseteq X$ such that $f \mid B$ is non-smooth and essentially injective. Fix a finer Polish topology on $X$ which generates the same Borel sets and with respect to which $B$ is clopen. Theorems 3.13 and 5.9 imply that there are Kakutani embeddings of $\sigma$ into $f \mid B$ and of $s_{[\mathbb{N}]}$ into $f \mid(X \backslash B)$ which are continuous with respect to the new topology, and the theorem follows.

We see next a maximality property of the shift:

Theorem 5.13. Suppose that $X$ is a Polish space and $f: X \rightarrow X$ is an aperiodic Borel function. Then the following are equivalent:

(1) The function $f$ is finite-to-one and well-founded.

(2) There is a Borel embedding of $f$ into $s_{[\mathbb{N}]}$.

(3) There is a Kakutani embedding of $f$ into $s_{[\mathbb{N}]}$.

Proof. It is clear that (2) $\Rightarrow(3) \Rightarrow(1)$, so we shall focus on (1) $\Rightarrow(2)$. We can assume, without loss of generality, that $X=2^{\mathbb{N}}$. Set $n(x)=\min \left\{n \in \mathbb{N}: f^{-n}(x)=\emptyset\right\}$, $k(x)=\left|f^{-1}(x)\right|$, and $X_{i}=\left\{x \in 2^{\mathbb{N}}: i<k(x)\right\}$. Fix Borel functions $f_{i}: X_{i} \rightarrow 2^{\mathbb{N}}$ such that $f^{-1}(x)=\left\{f_{i}(x): i<k(x)\right\}$ for all $x \in 2^{\mathbb{N}}$. Let $m(x)$ denote the unique natural number $m$ such that $x=f_{m} \circ f(x)$.

Set $\operatorname{Seq}(0)=\emptyset$ and define $\operatorname{Seq}(n+1)=\operatorname{Seq}(n) \cup\left(\mathbb{N} \times 2^{<\mathbb{N}} \times \operatorname{Seq}(n)^{<\mathbb{N}}\right)$. Put $\operatorname{Seq}=\bigcup_{n \in \mathbb{N}} \operatorname{Seq}(n)$. The restriction of an element of Seq to a natural number $i$ is defined recursively by $\left(m, s,\left\langle u_{l}\right\rangle_{l<k}\right) \mid i=\left(m, s \mid i,\left\langle u_{l} \mid i\right\rangle_{l<k}\right)$. We say that $v$ is an immediate predecessor of $u$ if $v=u_{l} \mid i$ for some $i \leq\left|u_{l}\right|$ and $l<k$, where $u=\left(m, s,\left\langle u_{l}\right\rangle_{l<k}\right)$. The predecessors of $u$ are defined recursively as the elements of Seq which are either immediate predecessors of $u$, or predecessors of $u_{l}$ for some $l<k$. As each element of Seq has only finitely many predecessors, there is an injection $\phi:$ Seq $\rightarrow \mathbb{N}$ such that $\phi(s) \leq \phi(t)$ whenever $s$ is a predecessor of $t$.

The well-foundedness of $f$ allows us to recursively define $\psi_{i}: 2^{\mathbb{N}} \rightarrow$ Seq by

$$
\psi_{i}(x)=\left(m(x), x \mid i,\left\langle\psi_{i} \circ f_{l}(x)\right\rangle_{l<k(x)}\right) .
$$

We claim that the function $\pi: 2^{\mathbb{N}} \rightarrow \mathbb{N}^{\mathbb{N}}$ given by

$$
[\pi(x)](i)=\phi \circ \psi_{n \circ f^{i}(x)} \circ f^{i}(x)
$$

is the desired embedding. To see this, note first that the definition of $\pi$ easily implies that $s_{[\mathbb{N}]} \circ \pi=\pi \circ f$, and if $i \leq j$, then $\psi_{n \circ f^{i}(x)} \circ f^{i}(x)$ is a predecessor of $\psi_{n \circ f^{j}(x)} \circ f^{j}(x)$, so $[\pi(x)](i) \leq[\pi(y)](j)$, thus $\pi\left(2^{\mathbb{N}}\right) \subseteq[\mathbb{N}]^{\mathbb{N}}$. 
It only remains to prove that $\pi$ is injective, for which it is sufficient to show that if $\pi(x)|(i+1)=\pi(y)|(i+1)$, then $x\left|n \circ f^{i}(x)=y\right| n \circ f^{i}(y)$, for $i \in \mathbb{N}$ and $x, y \in 2^{\mathbb{N}}$. We proceed by induction on $i$, simultaneously showing that $m \circ f^{j}(x)=m \circ f^{j}(y)$ for all $j \leq i$ and $x, y \in 2^{\mathbb{N}}$. The base case is clear, so suppose that $i \in \mathbb{Z}^{+}$, we have already established the claim strictly below $i$, and we are given $x, y \in 2^{\mathbb{N}}$ such that $\pi(x)|(i+1)=\pi(y)|(i+1)$. The fact that $[\pi(x)](i)=[\pi(y)](i)$ then ensures that $m \circ f^{i}(x)=m \circ f^{i}(y)$, so it only remains to show that $x\left|n \circ f^{i}(x)=y\right| n \circ f^{i}(y)$, for which it is clearly sufficient to show that $\psi_{n \circ f^{i}(x)}(x)=\psi_{n \circ f^{i}(y)}(y)$. Towards this end, we will inductively show that

$$
\forall j \leq i\left(\psi_{n \circ f^{i}(x)} \circ f^{j}(x)=\psi_{n \circ f^{i}(y)} \circ f^{j}(y)\right) .
$$

The base case $j=i$ follows trivially from the fact that $[\pi(x)](i)=[\pi(y)](i)$, so suppose that $j \in \mathbb{Z}^{+}$and we have already established the claim at $j$. As our original induction hypothesis ensures that $m \circ f^{j-1}(x)=m \circ f^{j-1}(y)$, it then follows that $\psi_{n \circ f^{i}(x)} \circ$ $f^{j-1}(x)=\psi_{n \circ f^{i}(y)} \circ f^{j-1}(y)$, which completes the proof.

The shift satisfies also a similar theorem for Kakutani reducibility:

Theorem 5.14. Suppose that $X$ is a Polish space and $f: X \rightarrow X$ is an aperiodic Borel function. Then the following are equivalent:

(1) The function $f$ is essentially both finite-to-one and well-founded.

(2) There is a Kakutani reduction of $f$ to $s_{[\mathbb{N}]}$.

Proof. To see (2) $\Rightarrow(1)$, simply note that if $f \leq_{K} s_{[\mathbb{N}]}$, then there is a complete, stable Borel set $B \subseteq X$ such that $f_{B} \sqsubseteq K s_{[\mathbb{N}]}$, thus $f_{B}$ is both finite-to-one and well-founded.

To see (1) $\Rightarrow(2)$, we first note the following fact:

Lemma 5.15. Suppose that $B \subseteq X$ is a recurrent Borel set such that $f_{B}$ is finite-to-one and well-founded. Then the set $C=[B] \vec{f}$ is Borel and the function $f_{C}$ is finite-to-one and well-founded.

Proof. The fact that $f_{B}$ is well-founded easily implies that $f_{C}$ is well-founded, and the fact that $f^{n} \mid B$ is finite-to-one for all $n \in \mathbb{N}$ ensures that $C$ is Borel. It remains to show that $f_{C}^{-1}(x)$ is finite for all $x \in C$. Towards this end, fix $i \in \mathbb{N}$ least such that $f^{i}(x) \in B$, fix $j \in \mathbb{N}$ such that $x \leq_{f} f^{j}(y)$ for all $y \in f_{B}^{-1} \circ f^{i}(x)$, and observe that $f_{C}^{-1}(x) \subseteq$ $\left\{f^{k}(y): k<j\right.$ and $\left.y \in f_{B}^{-1} \circ f^{i}(x)\right\}$.

Suppose that $f$ is essentially both finite-to-one and well-founded. Lemma 5.15 implies that there is a complete, stable Borel set $B \subseteq X$ such that $f_{B}$ is finite-to-one and wellfounded, so Theorem 5.13 ensures that $f_{B} \sqsubseteq_{K} s_{[\mathbb{N}]}$, thus $f \leq_{K} s_{[\mathbb{N}]}$.

We can now describe the Kakutani equivalence class of the increasing shift:

Theorem 5.16. All aperiodic, essentially both finite-to-one and well-founded, nonsmooth Borel functions on Polish spaces are Kakutani bi-reducible. Moreover, the class of such functions is closed under Kakutani equivalence. 
Proof. Suppose first that $X$ and $Y$ are Polish spaces and $f: X \rightarrow X$ and $g: Y \rightarrow Y$ are aperiodic, essentially both finite-to-one and well-founded, non-smooth Borel functions on Polish spaces. Theorem 5.14 ensures that $f \leq_{K} s_{[\mathbb{N}]}$ and Lemma 5.11 implies that $g$ is not essentially injective, so $s_{[\mathbb{N}]} \sqsubseteq_{K} g$ by Theorem 5.9, thus $f \leq_{K} g$.

Suppose now that $f$ and $g$ are Kakutani equivalent aperiodic Borel functions on Polish spaces. If $g$ is essentially both finite-to-one and well-founded, then Lemma 5.15 ensures that there is a complete, stable Borel set $B$ such that $g_{B}$ is finite-to-one and well-founded. As $f \approx_{K} g_{B}$, it follows that there is a complete, recurrent Borel set $A$ such that $f_{A}$ is finite-to-one and well-founded.

Theorem 5.17. All aperiodic Borel functions on Polish spaces which can be decomposed into an essentially injective, non-smooth part and an essentially both finite-to-one and well-founded, non-smooth part are Kakutani bi-reducible. Moreover, the class of such functions is closed under Kakutani equivalence.

Proof. This follows easily from Theorems 2.14 and 5.16

\section{Ranks on antichainable functions}

In this section, we provide a more detailed picture of the antichainable Borel functions under Kakutani equivalence, reducibility, and embeddability, with an emphasis on the functions of the form $s_{[\alpha]}$ and $s_{\langle\alpha\rangle}$.

It is clear that $s_{[\alpha]} \sqsubseteq_{B} s_{\langle\alpha\rangle}, s_{[\alpha]} \sqsubseteq_{B} s_{[\beta]}$, and $s_{\langle\alpha\rangle} \sqsubseteq_{B} s_{\langle\beta\rangle}$, for all limit ordinals $\alpha<\beta<\omega_{1}$. We shall eventually see that these inequalities are strict, even when Borel embeddability is replaced with Kakutani embeddability. In fact, the first of these is strict even when Borel embeddability is replaced with Kakutani reducibility. We shall now check that this is not literally the case for the latter two inequalities, although as we shall soon see, this is not so far from the truth.

Throughout this section, we use $\alpha+\beta, \alpha \cdot \beta$, and $\alpha^{\beta}$ to refer to the corresponding ordinal operations. An ordinal $\alpha$ is decomposable if there exist $\beta, \gamma<\alpha$ such that $\alpha=$ $\beta+\gamma$. An ordinal $\alpha$ is indecomposable if it is not decomposable, or equivalently, if it is isomorphic to all of its terminal segments. It is not difficult to show that $\omega^{\alpha}$ is the $\alpha^{\text {th }}$ indecomposable, non-zero ordinal.

Proposition 6.1. Suppose that $\alpha<\beta<\gamma<\omega_{1}$ are limit ordinals and $\gamma=\beta+\alpha$. Then $s_{[\gamma]} \leq_{K} s_{[\beta]}$ and $s_{\langle\gamma\rangle} \leq_{K} s_{\langle\beta\rangle}$.

Proof. We will show that $s_{[\gamma]} \leq_{K} s_{[\beta]}$, as the proof that $s_{\langle\gamma\rangle} \leq_{K} s_{\langle\beta\rangle}$ is identical. Define $[\gamma \backslash \beta]^{\mathbb{N}}=[\gamma]^{\mathbb{N}} \cap(\gamma \backslash \beta)^{\mathbb{N}}$, and observe that the set $A=[\beta]^{\mathbb{N}} \cup[\gamma \backslash \beta]^{\mathbb{N}}$ is complete and stable. Fix order-preserving injections $\phi: \beta \rightarrow \beta$ and $\psi: \gamma \backslash \beta \rightarrow \beta$ such that $\phi(\beta) \cap \psi(\gamma \backslash \beta)=\emptyset$, and define $\pi: A \rightarrow[\beta]^{\mathbb{N}}$ by

$$
[\pi(x)](n)= \begin{cases}\phi \circ x(n) & \text { if } x \in[\beta]^{\mathbb{N}}, \\ \psi \circ x(n) & \text { if } x \in[\gamma \backslash \beta]^{\mathbb{N}} .\end{cases}
$$

It is clear that $\pi$ is an embedding of $\left(s_{[\gamma]}\right)_{A}$ into $s_{[\beta]}$, thus $s_{[\gamma]} \leq_{K} s_{[\beta]}$. 
In order to show that Proposition 6.1 is the best possible result along these lines, it will be convenient to have at our disposal several invariants which come from classical ranks on trees. Recall that a tree on $\mathbb{N}$ is a set $T \subseteq \mathbb{N}<\mathbb{N}$ which is closed under initial segments. We use $*$ to denote the set of all such trees. Note that is a closed subset of $\mathcal{P}(\mathbb{N}<\mathbb{N})$, and therefore inherits a Polish topology.

A derivative on is a function $D: \rightarrow$ such that $D(T) \subseteq T$ and $T \subseteq U \Rightarrow$ $D(T) \subseteq D(U)$, for all $T, U \in$ * The iterates of a derivative $D$ are the functions $D^{\alpha}$ : $* \rightarrow$ defined recursively by $D^{0}(T)=T, D^{\alpha+1}(T)=D \circ D^{\alpha}(T)$ for $\alpha<\omega_{1}$, and $D^{\lambda}(T)=\bigcap_{\alpha<\lambda} D^{\alpha}(T)$ for limit ordinals $\lambda \leq \omega_{1}$.

The $D$-rank of a tree $T$, or $\rho_{D}(T)$, is the least ordinal $\alpha<\omega_{1}$ such that $D^{\alpha}(T)=$ $D^{\alpha+1}(T)$. The $D$-rank of a sequence $t \in T$ is given by

$$
\rho_{D}(T, t)= \begin{cases}\alpha & \text { if } t \in D^{\alpha}(T) \backslash D^{\alpha+1}(T), \\ \omega_{1} & \text { if } t \in D^{\omega_{1}}(T) .\end{cases}
$$

Note that if $D^{\omega_{1}}(T)=\emptyset$, then $\rho_{D}(T)=\rho_{D}(T, \emptyset)+1$.

Suppose now that $D$ is Borel, and set $\Omega_{D}=\left\{T \in D^{\omega_{1}}(T)=\varnothing\right\}$. Theorem 34.10 of [11] ensures that $\Omega_{D}$ is co-analytic and $\rho_{D}$ is a co-analytic rank on $\Omega_{D}$, thus Theorem 35.23 of [11] implies that if $A \subseteq \Omega_{D}$ is analytic, then $\sup _{T \in A} \rho_{D}(T)<\omega_{1}$.

The shift on $T$ is the function $s_{T}: T \backslash\{\emptyset\} \rightarrow T$ given by $s_{T}(t)=t \mid n$, where $n=|t|-1$. Let $¥(f, x)$ denote the analytic set of trees $T$ for which there is a Kakutani embedding $\pi$ of $s_{T}$ into $f$ such that $\pi(\emptyset) \leq_{f} x$. The $D$-rank of a point $x \in X$ is given by $\rho_{D}(f, x)=\sup \left\{\rho_{D}(T, \emptyset): T \in \nVdash(f, x)\right\}$. Note that the $D$-rank of a point is a local property, in the sense that $\rho_{D}(f, x)=\rho_{D}\left(f \mid[x]_{f}, x\right)$.

The $D$-rank of $f$ is given by $\rho_{D}(f)=\sup _{x \in X} \rho_{D}(f, x)$. The stable D-rank of $f$, or $\sigma_{D}(f)$, is the least ordinal $\alpha \leq \omega_{1}$ for which there is a complete, stable Borel set $B \subseteq X$ such that $\rho_{D}(f \mid B)=\alpha$.

The derivatives we consider will have the property that if $T$ and $U$ are trees and there is a Kakutani embedding of $s_{T}$ into $s_{U}$, then $\rho_{D}(T) \leq \rho_{D}(U)$. It is easy to see that if $D$ is such a derivative and $f \sqsubseteq_{K} g$, then $\rho_{D}(f) \leq \rho_{D}(g)$, thus $\rho_{D}$ is invariant under Kakutani bi-embeddability. Similarly, if $D$ is such a derivative and $f \leq_{K} g$, then $\sigma_{D}(f) \leq \sigma_{D}(g)$, thus $\sigma_{D}$ is invariant under Kakutani bi-reducibility. The Lusin-Novikov uniformization theorem easily implies that if $D$ is such a derivative, $f$ is countable-to-one, and $\rho_{D}(f)<\omega_{1}$, then the map $x \mapsto \rho_{D}(f, x)$ is Borel.

With these generalities out of the way, we are now ready to discuss the main two derivatives that we work with here, which are given by

$$
\mathrm{WF}(T)=\{t \in T: \exists u \in T(t \sqsubset u)\}
$$

and

$$
\mathrm{HI}(T)=\{t \in T: \exists u, v \in T(t \sqsubseteq u, v \quad \text { and } \quad u \perp v)\},
$$

where $u$ is incomparable with $v$, or $u \perp v$, if $u \nsubseteq v$ and $v \nsubseteq u$. We will also consider the modification $\mathrm{HI}^{\prime}$ of $\mathrm{HI}$ in which we ask for an infinite set of pairwise incomparable extensions. 
A sequence $x \in \mathbb{N}^{\mathbb{N}}$ is a branch of $T$ if $x \mid n \in T$ for all $n \in \mathbb{N}$, and a tree $T$ is perfect if every sequence in $T$ has a pair of incomparable extensions in $T$. It is easy to check that $\mathrm{WF}^{\omega_{1}}(T)=\emptyset$ if and only if $T$ does not have a branch, and $\mathrm{HI}^{\omega_{1}}(T)=\emptyset$ if and only if $T$ does not have a perfect subtree. It is also easy to see that $\mathrm{HI}^{\omega}(T) \subseteq \mathrm{HI}^{\prime}(T) \subseteq \mathrm{HI}(T)$, thus $\mathrm{HI}^{\omega_{1}}(T)=\left(\mathrm{HI}^{\prime}\right)^{\omega_{1}}(T)$.

We say that a function $f$ is hereditarily imperfect if there is no sequence $\left\langle x_{s}\right\rangle_{s \in 2^{<\mathbb{N}}}$ such that $s \sqsubseteq t \Rightarrow x_{t} \leq_{f} x_{s}$ and $s \perp t \Rightarrow x_{s} \perp_{f} x_{t}$, for all $s, t \in 2^{<\mathbb{N}}$. It is easy to see that $f$ is well-founded if and only if $\rho_{\mathrm{WF}}(f)<\omega_{1}$, and $f$ is hereditarily imperfect if and only if $\rho_{\mathrm{HI}}(f)<\omega_{1}$. It is also easy to see that $\rho_{\mathrm{HI}^{\prime}}(f) \leq \rho_{\mathrm{HI}}(f) \leq \omega \cdot \rho_{\mathrm{HI}^{\prime}}(f)$.

Proposition 6.2. Suppose that $\alpha<\omega_{1}$ is a limit ordinal, $\beta<\omega_{1}, k<\omega, x \in[\alpha]^{\mathbb{N}}$, $y \in\langle\alpha\rangle^{\mathbb{N}}$, and $x(0)=\omega \cdot \beta+k$. Then $\rho_{\mathrm{WF}}\left(s_{[\alpha]}, x\right)=x(0), \rho_{\mathrm{HI}}\left(s_{[\alpha]}, x\right)=\omega \cdot \beta+\lfloor k / 2\rfloor$, $\rho_{\mathrm{HI}^{\prime}}\left(s_{[\alpha]}, x\right)=\beta, \rho_{\mathrm{WF}}\left(s_{\langle\alpha\rangle}, y\right)=\omega_{1}$, and $\rho_{\mathrm{HI}}\left(s_{\langle\alpha\rangle}, y\right)=\rho_{\mathrm{HI}^{\prime}}\left(s_{\langle\alpha\rangle}, y\right)=y(0)$.

Proof. It is clear that $\rho_{\mathrm{WF}}\left(s_{\langle\alpha\rangle}, y\right)=\omega_{1}$. As the proofs of the remaining statements are essentially the same, we shall only prove that $\rho_{\mathrm{WF}}\left(s_{[\alpha]}, x\right)=x(0)$. We proceed by transfinite induction on $x(0)$. It is clear that if $x(0)=0$, then $\rho_{\mathrm{WF}}\left(s_{[\alpha]}, x\right)=0$. Suppose now that we have already established the lemma for all $x \in[\alpha]^{\mathbb{N}}$ with $x(0)<\beta$, and observe that if $x \in[\alpha]^{\mathbb{N}}$ and $x(0)=\beta$, then the induction hypothesis ensures that $\rho_{\mathrm{WF}}\left(s_{[\alpha]}, x\right)=\sup _{\gamma<\beta} \rho_{\mathrm{WF}}\left(s_{[\alpha]}, \gamma x\right)+1=\sup _{\gamma<\beta} \gamma+1=\beta$.

As an immediate corollary, we obtain the following:

Proposition 6.3. Suppose that $\alpha, \beta<\omega_{1}$ and $\alpha=\omega \cdot \beta$. Then $\rho_{\mathrm{WF}}\left(s_{[\alpha]}\right)=\rho_{\mathrm{HI}}\left(s_{[\alpha]}\right)=$ $\rho_{\mathrm{HI}}\left(s_{\langle\alpha\rangle}\right)=\rho_{\mathrm{HI}^{\prime}}\left(s_{\langle\alpha\rangle}\right)=\alpha, \rho_{\mathrm{HI}^{\prime}}\left(s_{[\alpha]}\right)=\beta$, and $\rho_{\mathrm{WF}}\left(s_{\langle\alpha\rangle}\right)=\omega_{1}$.

While Propositions 6.1 and 6.3 together rule out the analog of Proposition 6.3 for stable ranks at decomposable ordinals, we do have the next best thing:

Proposition 6.4. Suppose that $\alpha, \gamma<\omega_{1}$ are limit ordinals, $\beta<\omega_{1}$, and $\alpha=\omega \cdot \beta$ is indecomposable. Then $\sigma_{\mathrm{WF}}\left(s_{[\alpha]}\right)=\sigma_{\mathrm{HI}}\left(s_{[\alpha]}\right)=\sigma_{\mathrm{HI}}\left(s_{\langle\alpha\rangle}\right)=\sigma_{\mathrm{HI}^{\prime}}\left(s_{\langle\alpha\rangle}\right)=\alpha, \sigma_{\mathrm{HI}^{\prime}}\left(s_{[\alpha]}\right)=$ $\beta$, and $\sigma_{\mathrm{WF}}\left(s_{\langle\gamma\rangle}\right)=\omega_{1}$.

Proof. We shall only prove that $\sigma_{\mathrm{WF}}\left(s_{[\alpha]}\right)=\alpha$, as the other proofs are similar.

Lemma 6.5. Suppose that $B \subseteq[\alpha]^{\mathbb{N}}$ is a complete, stable Borel set. Then there exists $\delta<\alpha$ such that $[\alpha \backslash \delta]^{\mathbb{N}} \cap[x]_{s_{[\alpha]}} \subseteq B$ for comeagerly many $x \in[\alpha]^{\mathbb{N}}$.

Proof. As $s_{[\alpha]}$ is a continuous, open map and $[\alpha]^{\mathbb{N}}=\bigcup_{n \in \mathbb{N}} s_{[\alpha]}^{-n}(B)$, it follows that $B$ is non-meager in $[\alpha]^{\mathbb{N}}$, so there exist $n \in \mathbb{N}$ and a strictly increasing sequence $t \in \alpha^{n}$ such that $B$ is comeager in $[\alpha]^{\mathbb{N}} \cap \mathcal{N}_{t}$. Fix $\delta<\alpha$ such that $\delta>t(i)$ for all $i<n$. The fact that $s_{[\alpha]}$ is continuous and open implies that $s_{[\alpha]}^{n}(B)$ is comeager in $[\alpha \backslash \delta]^{\mathbb{N}}$. As $B$ is stable, it follows that the set $M=\left[[\alpha \backslash \delta]^{\mathbb{N}} \backslash B\right]_{s_{[\alpha]}}$ is meager, and it is clear that if $x \notin M$, then $[\alpha \backslash \delta]^{\mathbb{N}} \cap[x]_{s_{[\alpha]}} \subseteq B$. 
Suppose now that $B \subseteq[\alpha]^{\mathbb{N}}$ is a complete, stable Borel set. Noting that the set $C=$ $\left\{x \in[\alpha]^{\mathbb{N}}: \alpha=\sup _{n \in \mathbb{N}} x(n)\right\}$ is comeager in $[\alpha]^{\mathbb{N}}$, it follows from Lemma 6.5 that there exists $x \in C$ such that $[\alpha \backslash \delta]^{\mathbb{N}} \cap[x]_{s_{[\alpha]}} \subseteq B$. The fact that $\alpha$ is indecomposable ensures that there is an order-preserving bijection $\phi: \alpha \backslash \delta \rightarrow \alpha$, and Proposition 6.3 then implies that $\rho_{\mathrm{WF}}\left(s_{[\alpha]} \mid B\right) \geq \sup _{n \in \mathbb{N}} \rho_{\mathrm{WF}}\left(s_{[\alpha]} \mid[\alpha \backslash \delta]^{\mathbb{N}}, s_{[\alpha]}^{n}(x)\right)=\sup _{n \in \mathbb{N}} \phi \circ x(n)=\alpha$, thus $\sigma_{\mathrm{WF}}\left(s_{[\alpha]}\right)=\alpha$.

Remark 6.6. Propositions 6.1 and 6.4 imply that the stable ranks of $s_{[\alpha]}$ and $s_{\langle\alpha\rangle}$ are indecomposable. In fact, a straightforward modification of the proof of Proposition 6.1 shows that if $f$ is any aperiodic, countable-to-one Borel function on a Polish space, then $\sigma_{\mathrm{WF}}(f), \sigma_{\mathrm{HI}}(f)$, and $\sigma_{\mathrm{HI}^{\prime}}(f)$ are indecomposable.

As an immediate consequence of Proposition 6.4, we obtain the following:

Proposition 6.7. Suppose that $\alpha<\beta<\omega_{1}$ are indecomposable limit ordinals and $\gamma, \delta<\omega_{1}$ are limit ordinals. Then $s_{[\beta]} \bigsqcup_{K} s_{\langle\alpha\rangle}$ and $s_{\langle\gamma\rangle} \bigsqcup_{K} s_{[\delta]}$.

Remark 6.8. An identical argument can be used to establish the analog of Proposition 6.7 in which the notion of Kakutani reducibility is weakened by removing the requirement of injectivity, since stable ranks are invariant under such maps.

Next, we will show that every aperiodic, countable-to-one, well-founded Borel function is Borel embeddable into $s_{[\alpha]}$ for some $\alpha<\omega_{1}$. Perhaps the most natural way of proving such a theorem would be to show that $s_{[\alpha]}$ is universal among the aperiodic, countable-to-one Borel functions $f$ for which $\rho_{\mathrm{WF}}(f) \leq \alpha$. However, the following observation shows that this is false:

Proposition 6.9. Suppose that $\beta<\alpha<\omega_{1}$ and $\alpha=\omega \cdot \beta$. Then $\rho_{\mathrm{WF}}\left(s_{[\alpha]} \times s_{\mathbb{N}}\right)=\alpha$, but $s_{[\alpha]} \times s_{\mathbb{N}} \not_{K} s_{[\alpha]}$.

Proof. Simply note that the proofs of Propositions 6.3 and 6.4 imply that $\rho_{\mathrm{WF}}\left(s_{[\alpha]} \times\right.$ $\left.s_{\mathbb{N}}\right)=\sigma_{\mathrm{HI}^{\prime}}\left(s_{[\alpha]} \times s_{\mathbb{N}}\right)=\alpha$, while Proposition 6.4 ensures that $\sigma_{\mathrm{HI}^{\prime}}\left(s_{[\alpha]}\right)=\beta$.

Nevertheless, we do have the following:

Proposition 6.10. Suppose that $\alpha<\omega_{1}$ is a limit ordinal. Then $s_{[\alpha]} \times s_{\mathbb{N}} \sqsubseteq_{B} s_{[\omega \cdot \alpha]}$.

Proof. Define $\pi:[\alpha]^{\mathbb{N}} \times \mathbb{N}^{\mathbb{N}} \rightarrow[\omega \cdot \alpha]^{\mathbb{N}}$ by $[\pi(x, y)](n)=\omega \cdot x(n)+y(n)$. It is clear that $\pi$ is injective, and if $(x, y) \in[\alpha]^{\mathbb{N}} \times \mathbb{N}^{\mathbb{N}}$, then

$$
\begin{aligned}
s_{[\omega \cdot \alpha]} \circ \pi(x, y) & =s_{[\omega \cdot \alpha]}\left(\langle\omega \cdot x(n)+y(n)\rangle_{n \in \mathbb{N}}\right)=\langle\omega \cdot x(n+1)+y(n+1)\rangle_{n \in \mathbb{N}} \\
& =\pi \circ\left(s_{[\alpha]} \times s_{\mathbb{N}}\right)(x, y),
\end{aligned}
$$

thus $\pi$ is an embedding of $s_{[\alpha]} \times s_{\mathbb{N}}$ into $s_{[\omega \cdot \alpha]}$.

Proposition 6.11. Suppose that $\alpha<\omega_{1}$ is a limit ordinal. Then $s_{[\alpha]} \times s_{\mathbb{N}} \sqsubseteq_{K} s_{\langle\alpha\rangle}$. 
Proof. It is sufficient to show that $s_{[\alpha]} \times s_{\mathbb{N}} \cong_{B}\left(s_{\langle\alpha\rangle}\right)_{B}$, where $B=\left\{x \in\langle\alpha\rangle^{\mathbb{N}}: x(0)<\right.$ $x(1)\}$. To this end, define $\pi:[\alpha]^{\mathbb{N}} \times \mathbb{N}^{\mathbb{N}} \rightarrow B$ by $\pi(x, y)=x(0) \oplus \bigoplus_{n \in \mathbb{N}} x(n+1)^{y(n)+1}$, where $x(n+1)^{y(n)+1}$ denotes the constant sequence of length $y(n)+1$ with value $x(n+1)$. Clearly $\pi$ is bijective, and if $(x, y) \in[\alpha]^{\mathbb{N}} \times \mathbb{N}^{\mathbb{N}}$, then

$$
\begin{aligned}
\left(s_{\langle\alpha\rangle}\right)_{B} \circ \pi(x, y) & =\left(s_{\langle\alpha\rangle}\right)_{B}\left(x(0) \oplus \bigoplus_{n \in \mathbb{N}} x(n+1)^{y(n)+1}\right) \\
& =x(1) \oplus \bigoplus_{n \in \mathbb{N}} x(n+2)^{y(n+1)+1}=\pi \circ\left(s_{[\alpha]} \times s_{\mathbb{N}}\right)(x, y),
\end{aligned}
$$

thus $\pi$ is an isomorphism of $s_{[\alpha]} \times s_{\mathbb{N}}$ and $\left(s_{\langle\alpha\rangle}\right)_{B}$.

We will now establish that $s_{[\alpha]} \times s_{\mathbb{N}}$ does have the desired universality property, which implies our earlier claim that every aperiodic, countable-to-one, well-founded Borel function is Borel embeddable into $s_{[\alpha]}$, for some $\alpha<\omega_{1}$.

Theorem 6.12. Suppose that $\alpha<\omega_{1}$ is a limit ordinal, $X$ is a Polish space, and $f$ : $X \rightarrow X$ is an aperiodic, countable-to-one Borel function. Then the following are equivalent:

(1) The WF-rank of $f$ is at most $\alpha$.

(2) There is a Borel embedding of $f$ into $s_{[\alpha]} \times s_{\mathbb{N}}$.

(3) There is a Kakutani embedding of $f$ into $s_{[\alpha]} \times s_{\mathbb{N}}$.

As a consequence, the following are also equivalent:

(a) The stable WF-rank of $f$ is at most $\alpha$.

(b) There is a Kakutani reduction of $f$ into $s_{[\alpha]} \times s_{\mathbb{N}}$.

Proof. We will only prove (1) $\Rightarrow(2)$, as the rest of the theorem easily follows. Fix a Borel generator $\chi: X \rightarrow \mathbb{N}$, and define $\pi: X \rightarrow[\alpha]^{\mathbb{N}} \times \mathbb{N}^{\mathbb{N}}$ by

$$
[\pi(x)](n)=\left(\rho_{\mathrm{WF}}\left(f, f^{n}(x)\right), \chi \circ f^{n}(x)\right) .
$$

The fact that $\chi$ is a generator ensures that $\pi$ is injective, and if $x \in X$, then

$$
\begin{aligned}
\left(s_{[\alpha]} \times s_{\mathbb{N}}\right) \circ \pi(x) & =\left(s_{[\alpha]} \times s_{\mathbb{N}}\right)\left(\left\langle\left(\rho_{\mathrm{WF}}\left(f, f^{n}(x)\right), \chi \circ f^{n}(x)\right)\right\rangle_{n \in \mathbb{N}}\right) \\
& =\left\langle\left(\rho_{\mathrm{WF}}\left(f, f^{n+1}(x)\right), \chi \circ f^{n+1}(x)\right)\right\rangle_{n \in \mathbb{N}}=\pi \circ f(x),
\end{aligned}
$$

thus $\phi$ is an embedding of $f$ into $s_{[\alpha]} \times s_{\mathbb{N}}$.

Along similar lines, we would next like to show that every aperiodic, countable-to-one, hereditarily imperfect Borel function is Borel embeddable into $s_{\langle\alpha\rangle}$ for some $\alpha<\omega_{1}$. Unfortunately, this is false, as such functions need not be antichainable. However, we do have the following:

Proposition 6.13. Suppose that $X$ is a Polish space and $f: X \rightarrow X$ is an aperiodic, countable-to-one, hereditarily imperfect Borel function. Then $f$ can be decomposed into antichainable and essentially injective parts. 
Proof. Define $A=\left\{x \in X: \forall n \in \mathbb{N} \exists m \geq n\left(\rho_{\mathrm{HI}}\left(f, f^{n}(x)\right)<\rho_{\mathrm{HI}}\left(f, f^{m}(x)\right)\right)\right\}$. It is clear that $A$ is an invariant Borel set. For each $\alpha<\rho_{\mathrm{HI}}(f)$ and $n \in \mathbb{N}$, let $A_{\alpha n}$ denote the set of all $x \in A$ for which $\rho_{\mathrm{HI}}(f, x)=\alpha$ and $n$ is minimal with the property that $\alpha<\rho_{\mathrm{HI}}\left(f, f^{n}(x)\right)$. It is clear that these sets are antichains whose union is $A$, thus $f \mid A$ is antichainable.

Set $B=X \backslash A$ and $C=\left\{x \in B: \forall n \in \mathbb{N}\left(\rho_{\mathrm{HI}}(f, x)=\rho_{\mathrm{HI}}\left(f, f^{n}(x)\right)\right)\right\}$. Clearly $C$ is an $(f \mid B)$-complete, stable Borel set. Now observe that if $x, y \in C$ are distinct and $z=f(x)=f(y)$, then $\rho_{\mathrm{HI}}(f, z)>\rho_{\mathrm{HI}}(f, x)=\rho_{\mathrm{HI}}(f, y)$, which contradicts the definition of $C$, so $f \mid C$ is injective, thus $f \mid B$ is essentially injective.

Let $d$ denote the function from $\mathbb{Z}^{+} \times \mathbb{N}^{\mathbb{N}}$ to $\mathbb{N}^{\mathbb{N}}$ given by

$$
[d(x)](n)= \begin{cases}x(0)-1 & \text { if } n=0, \\ x(n) & \text { otherwise }\end{cases}
$$

Given an aperiodic Borel function $f: X \rightarrow X$, define $f^{\prime}: X \times \mathbb{N}^{\mathbb{N}} \rightarrow X \times \mathbb{N}^{\mathbb{N}}$ by

$$
f^{\prime}(x, y)= \begin{cases}\left(f(x), s_{\mathbb{N}}(y)\right) & \text { if } y(0)=0, \\ (x, d(y)) & \text { otherwise. }\end{cases}
$$

A straightforward induction shows that $\rho_{\mathrm{HI}}\left(f^{\prime}\right)=\rho_{\mathrm{WF}}(f)$. John Clemens initially suggested $f^{\prime}$ to us as a natural modification of $f$ whose stable WF-rank is $\omega_{1}$.

Proposition 6.14. Suppose that $\alpha<\omega_{1}$ is a limit ordinal. Then $s_{[\alpha]}^{\prime} \cong_{B} s_{\langle\alpha\rangle}$.

Proof. Define $\pi:[\alpha]^{\mathbb{N}} \times \mathbb{N}^{\mathbb{N}} \rightarrow\langle\alpha\rangle^{\mathbb{N}}$ by $\pi(x, y)=\bigoplus_{n \in \mathbb{N}} x(n)^{y(n)+1}$, where $x(n)^{y(n)+1}$ denotes the constant sequence of length $y(n)+1$ with value $x(n)$. Clearly $\pi$ is bijective, and if $(x, y) \in[\alpha]^{\mathbb{N}} \times \mathbb{N}^{\mathbb{N}}$, then

$$
\begin{aligned}
s_{\langle\alpha\rangle} \circ \pi(x, y) & =s_{\langle\alpha\rangle}\left(\bigoplus_{n \in \mathbb{N}} x(n)^{y(n)+1}\right)=x(0)^{y(0)} \oplus \bigoplus_{n \in \mathbb{N}} x(n+1)^{y(n+1)+1} \\
& =\pi \circ s_{[\alpha]}^{\prime}(x, y),
\end{aligned}
$$

thus $\pi$ is an isomorphism of $s_{[\alpha]}^{\prime}$ and $s_{\langle\alpha\rangle}$.

Proposition 6.15. Suppose that $\alpha<\omega_{1}$ is a limit ordinal. Then $\left(s_{[\alpha]} \times s_{\mathbb{N}}\right)^{\prime} \sqsubseteq_{B} s_{\langle\omega \cdot \alpha\rangle}$.

Proof. By Proposition 6.10 there is a Borel embedding of $s_{[\alpha]} \times s_{\mathbb{N}}$ into $s_{[\omega \cdot \alpha]}$. As any such map clearly induces a Borel embedding of $\left(s_{[\alpha]} \times s_{\mathbb{N}}\right)^{\prime}$ into $s_{[\omega \cdot \alpha]}^{\prime}$, the desired result follows from Proposition 6.14 .

Theorem 6.16. Suppose that $\alpha<\omega_{1}$ is a limit ordinal, $X$ is a Polish space, and $f: X \rightarrow$ $X$ is an antichainable, aperiodic, countable-to-one Borel function. Then the following are equivalent:

(1) The HI-rank of $f$ is at most $\alpha$.

(2) There is a Borel embedding of $f$ into $\left(s_{[\alpha]} \times s_{\mathbb{N}}\right)^{\prime}$.

(3) There is a Kakutani embedding of $f$ into $\left(s_{[\alpha]} \times s_{\mathbb{N}}\right)^{\prime}$.

As a consequence, the following are also equivalent:

(a) The stable HI-rank of $f$ is at most $\alpha$.

(b) There is a Kakutani reduction of $f$ into $\left(s_{[\alpha]} \times s_{\mathbb{N}}\right)^{\prime}$. 
Proof. We will again prove only $(1) \Rightarrow(2)$, as the rest of the theorem easily follows. Set $A=\left\{x \in X: \rho_{\mathrm{HI}}(f, x)<\rho_{\mathrm{HI}}(f, f(x))\right\}$ and $B=\{x \in X: \forall n \in \mathbb{N} \exists m \geq$ $\left.n\left(f^{m}(x) \in A\right)\right\}$. The proof of Proposition 6.13 easily implies that the restriction of $f$ to the complement of $B$ is essentially injective, thus smooth (by Lemma 5.11), and this easily implies that it is Borel embeddable into $\left(s_{[\alpha]} \times s_{\mathbb{N}}\right)^{\prime}$. It is therefore sufficient to show that $f_{B} \sqsubseteq B\left(s_{[\alpha]} \times s_{\mathbb{N}}\right)^{\prime}$.

For each $x \in B$, let $k_{0}(x)$ denote the least $k \in \mathbb{N}$ such that $f^{k}(x) \in A$, and let $k_{n+1}(x)$ denote the least $k>k_{n}(x)$ such that $f^{k}(x) \in A$. Fix a Borel $f_{A \cap B}$-generator $\chi: A \rightarrow \mathbb{N}$, and define $l: X \rightarrow \mathbb{N}^{\mathbb{N}}$ by

$$
[l(x)](n)= \begin{cases}k_{0}(x) & \text { if } n=0, \\ k_{n}(x)-k_{n-1}(x)-1 & \text { otherwise }\end{cases}
$$

Finally, define $\pi: X \rightarrow[\alpha]^{\mathbb{N}} \times \mathbb{N}^{\mathbb{N}} \times \mathbb{N}^{\mathbb{N}}$ by

$$
\pi(x)=\left(\left\langle\rho_{\mathrm{HI}}\left(f, f^{k_{n}(x)}(x)\right)\right\rangle_{n \in \mathbb{N}},\left\langle\chi \circ f^{k_{n}(x)}(x)\right\rangle_{n \in \mathbb{N}}, l(x)\right) .
$$

To see that $\pi$ is injective, suppose that $\pi(x)=\pi(y)$. The fact that $\chi$ is a generator then implies that $f^{k_{0}(x)}(x)=f^{k_{0}(y)}(y)$. Let $z$ denote this common iterate, and observe that the definition of $k_{0}(x)$ ensures that $\rho_{\mathrm{HI}}(x)=\rho_{\mathrm{HI}}(y)=\rho_{\mathrm{HI}}(z)$. This implies that $x \leq_{f} y$ or $y \leq_{f} x$, and since $k_{0}(x)=k_{0}(y)$, it follows that $x=y$.

Observe now that if $x \in B$ and $k_{0}(x)=0$, then

$$
\begin{aligned}
\left(s_{[\alpha]} \times s_{\mathbb{N}}\right)^{\prime} \circ \pi(x) & =\left(s_{[\alpha]} \times s_{\mathbb{N}}\right)^{\prime}\left(\left\langle\rho_{\mathrm{HI}}\left(f, f^{k_{n}(x)}(x)\right)\right\rangle_{n \in \mathbb{N}},\left\langle\chi \circ f^{k_{n}(x)}(x)\right\rangle_{n \in \mathbb{N}}, l(x)\right) \\
& =\left(\left\langle\rho_{\mathrm{HI}}\left(f, f^{k_{n+1}(x)}(x)\right)\right\rangle_{n \in \mathbb{N}},\left\langle\chi \circ f^{k_{n+1}(x)}(x)\right\rangle_{n \in \mathbb{N}}, s_{\mathbb{N}} \circ l(x)\right) \\
& =\pi \circ f(x) .
\end{aligned}
$$

Similarly, if $x \in B$ and $k_{0}(x)>0$, then

$$
\begin{aligned}
\left(s_{[\alpha]} \times s_{\mathbb{N}}\right)^{\prime} \circ \pi(x) & =\left(s_{[\alpha]} \times s_{\mathbb{N}}\right)^{\prime}\left(\left\langle\rho_{\mathrm{HI}}\left(f, f^{k_{n}(x)}(x)\right)\right\rangle_{n \in \mathbb{N}},\left\langle\chi \circ f^{k_{n}(x)}(x)\right\rangle_{n \in \mathbb{N}}, l(x)\right) \\
& =\left(\left\langle\rho_{\mathrm{HI}}\left(f, f^{k_{n}(x)}(x)\right)\right\rangle_{n \in \mathbb{N}},\left\langle\chi \circ f^{k_{n}(x)}(x)\right\rangle_{n \in \mathbb{N}}, d \circ l(x)\right) \\
& =\pi \circ f(x),
\end{aligned}
$$

thus $\pi$ is an embedding of $f_{B}$ into $\left(s_{[\alpha]} \times s_{\mathbb{N}}\right)^{\prime}$.

It is important to note that if the definition of Kakutani reducibility is relaxed in such a fashion that we can freely go down to complete, recurrent Borel sets, then much of the hierarchy we have described in this section collapses:

Proposition 6.17. Suppose that $X$ is a Polish space and $f: X \rightarrow X$ is an antichainable, aperiodic, countable-to-one Borel function. Then there is a complete, recurrent Borel set $B \subseteq X$ such that $\rho_{\mathrm{WF}}\left(f_{B}\right)=\omega$, thus $f_{B} \sqsubseteq B s_{[\mathbb{N}]} \times s_{\mathbb{N}}$.

Proof. By Theorem 3.13, there is a decreasing sequence $\left\langle A_{n}\right\rangle_{n \in \mathbb{N}}$ of complete, stable Borel sets with empty intersection. Set $B_{n}=f^{-1}\left(A_{n}\right) \backslash A_{n}$, and observe that if $x \in B_{m}$, $y \in B_{n}$, and $x<_{f} y$, then $m<n$. Setting $B=\bigcup_{n \in \mathbb{N}} B_{n}$, it follows that $\rho_{\mathrm{WF}}\left(f_{B}\right)=\omega$, thus Theorem 6.12 ensures that $f_{B} \sqsubseteq B s_{[\mathbb{N}]} \times s_{\mathbb{N}}$. 


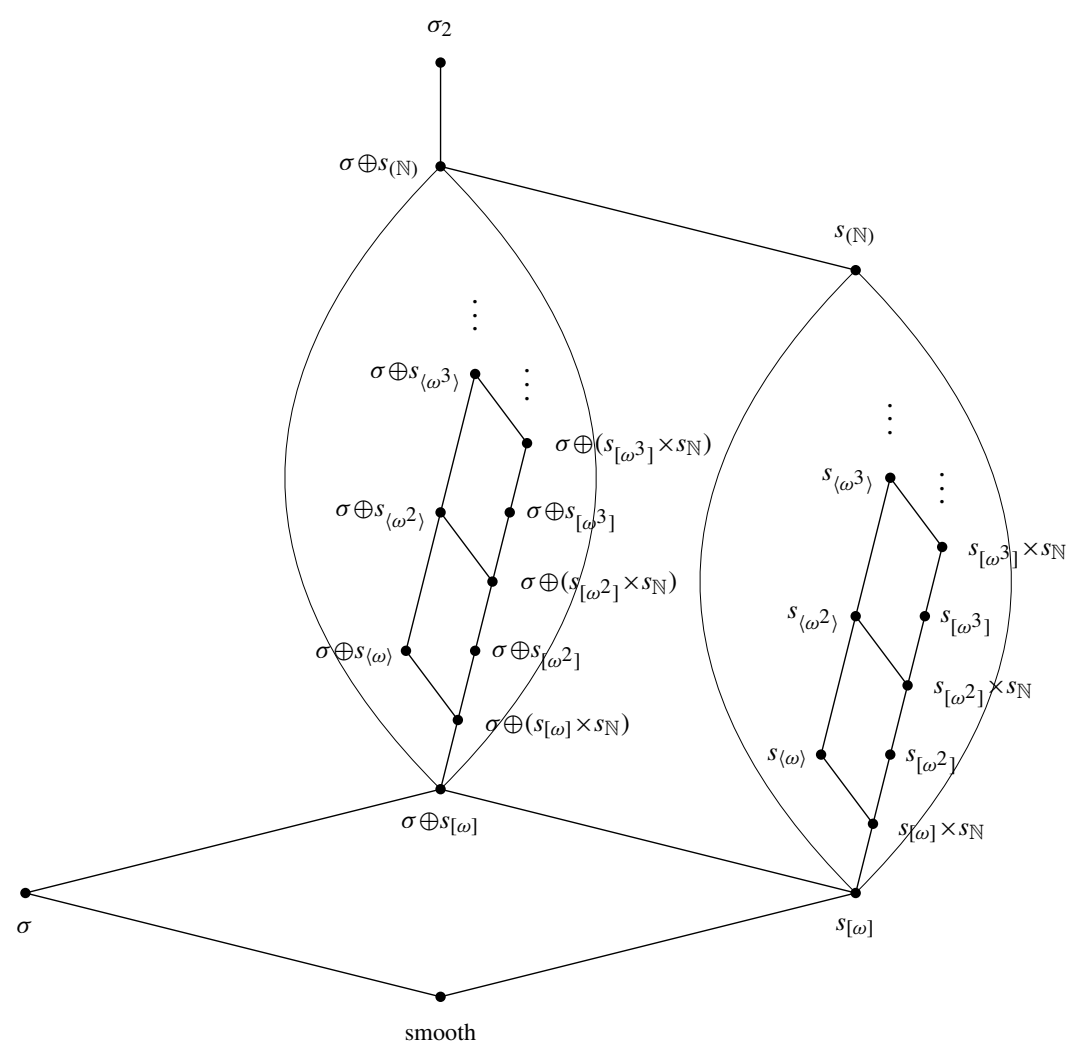

Fig. 5. Kakutani reducibility of countable-to-one Borel functions.

We can now verify that Kakutani equivalence trivializes above $s_{[\mathbb{N}]} \times s_{\mathbb{N}}$ :

Theorem 6.18. All antichainable, aperiodic, essentially countable-to-one Borel functions on Polish spaces to which $s_{[\mathbb{N}]} \times s_{\mathbb{N}}$ is Kakutani reducible are Kakutani equivalent. Moreover, the class of such functions is closed under Kakutani bi-reducibility.

Proof. Suppose first that $X$ and $Y$ are Polish spaces and $f: X \rightarrow X$ and $g: Y \rightarrow Y$ are antichainable, aperiodic, essentially countable-to-one Borel functions to which $s_{[\mathbb{N}]} \times s_{\mathbb{N}}$ is Kakutani reducible. Proposition 6.17 implies that there are complete, recurrent Borel sets $A \subseteq X$ and $B \subseteq Y$ and Kakutani embeddings $\phi_{A}: A \rightarrow[\mathbb{N}]^{\mathbb{N}} \times \mathbb{N}^{\mathbb{N}}$ and $\phi_{B}$ : $B \rightarrow[\overline{\mathbb{N}}]^{\mathbb{N}} \times \mathbb{N}^{\mathbb{N}}$ of $f_{A}$ and $g_{B}$ into $s_{[\mathbb{N}]} \times s_{\mathbb{N}}$. Fix complete, stable Borel sets $C, D \subseteq$ $[\mathbb{N}]^{\mathbb{N}} \times \mathbb{N}^{\mathbb{N}}$ such that $\left(s_{[\mathbb{N}]} \times s_{\mathbb{N}}\right)_{C} \sqsubseteq K f$ and $\left(s_{[\mathbb{N}]} \times s_{\mathbb{N}}\right)_{D} \sqsubseteq_{K} g$, and note that the sets $A^{\prime}=A \cap \phi_{A}^{-1}(D)$ and $B^{\prime}=B \cap \phi_{B}^{-1}(C)$ are complete and recurrent. As $f_{A^{\prime}} \sqsubseteq_{K} g$ and $g_{B^{\prime}} \sqsubseteq_{K} f$, Lemma 2.13 ensures that $f \approx_{K} g$.

Suppose now that $f$ and $g$ are Kakutani bi-reducible aperiodic Borel functions on Polish spaces. The fact that $\leq_{K}$ is a quasi-order ensures that $s_{[\mathbb{N}]} \times s_{\mathbb{N}} \leq_{K} f$ if and only 
if $s_{[\mathbb{N}]} \times s_{\mathbb{N}} \leq_{K} g$. It is clear that $f$ is antichainable and essentially countable-to-one if and only if $g$ is antichainable and essentially countable-to-one.

Theorem 6.19. All aperiodic, essentially countable-to-one Borel functions on Polish spaces which can be decomposed into an antichainable part to which $s_{[\mathbb{N}]} \times s_{\mathbb{N}}$ is Kakutani reducible and an essentially injective, non-smooth part are Kakutani equivalent. Moreover, the class of such functions is closed under Kakutani bi-reducibility.

Proof. This follows easily from Theorems 2.14 and 6.18

Finally, we check that the hierarchy does not collapse completely:

Proposition 6.20. The function $s_{[\mathbb{N}]} \times s_{\mathbb{N}}$ is not essentially finite-to-one.

Proof. Suppose, towards a contradiction, that $A \subseteq[\mathbb{N}]^{\mathbb{N}} \times \mathbb{N}^{\mathbb{N}}$ is a complete, recurrent Borel set such that $\left(s_{[\mathbb{N}]} \times s_{\mathbb{N}}\right)_{A}$ is essentially finite-to-one. By Lemma 5.15, we can assume that $A$ is stable, so $\rho_{\mathrm{HI}^{\prime}}\left(\left(s_{[\mathbb{N}]} \times s_{\mathbb{N}}\right)_{A}\right)=0$, which contradicts the fact that $\sigma_{\mathrm{HI}^{\prime}}\left(s_{[\mathbb{N}]} \times s_{\mathbb{N}}\right)=\omega$.

We close this section by noting that $s_{[\alpha]} \times s_{\mathbb{N}}$ and $s_{[\alpha]} \times s_{(\mathbb{N})}$ are Borel bi-embeddable, thus our results remain true if we replace the former with the latter.

\section{Kakutani equivalence of countable-to-one functions}

We begin this section with a summary of our knowledge of Kakutani equivalence:

Theorem 7.1. Suppose that $X$ is a Polish space and $f: X \rightarrow X$ is an aperiodic, essentially countable-to-one, non-smooth Borel function on a Polish space which is not Kakutani equivalent to one of the following functions:

(1) The odometer $\sigma$.

(2) The increasing shift $s_{[\mathbb{N}]}$.

(3) The disjoint sum $\sigma \oplus s_{[\mathbb{N}]}$.

(4) The 2-to-1 analog of the odometer $\sigma_{2}$.

(5) The injective shift $s_{(\mathbb{N})}$.

(6) The disjoint sum $\sigma \oplus s_{(\mathbb{N})}$.

Then $f$ can be decomposed into a part which is essentially injective and a part which is essentially strictly $\leq_{K}$-between $s_{[\mathbb{N}]}$ and $s_{[\mathbb{N}]} \times s_{(\mathbb{N})}$.

Proof. Clearly we can assume that $f$ is countable-to-one. By Theorem 4.5, our assumption that $f \not_{K} \quad \sigma_{2}$ ensures that $f$ is of the form $f_{a} \oplus f_{i}$, where $f_{a}$ is antichainable and $f_{i}$ is essentially injective. Note that $f_{a}$ cannot be essentially injective, since otherwise $f$ is essentially injective, in which case Theorem 2.14 ensures that $f \approx_{K} \sigma$. As Theorem 2.14 ensures that $f_{i}$ is either smooth or Kakutani equivalent to $\sigma$, we can clearly assume that $f$ is antichainable and non-essentially injective. By Proposition 6.17, there is a complete, recurrent Borel set $B \subseteq X$ such that $f_{B} \sqsubseteq_{B} s_{[\mathbb{N}]} \times s_{(\mathbb{N})}$. Theorem 5.9 implies that $s_{[\mathbb{N}]} \sqsubseteq_{K} f_{B}$, and it follows that $s_{[\mathbb{N}]}<_{K} f_{B}$, since otherwise Theorem 5.16 ensures that 
$f \approx_{K} s_{[\mathbb{N}]}$. Finally, observe that $f_{B}<_{K} s_{[\mathbb{N}]} \times s_{(\mathbb{N})}$, since otherwise Theorem 6.18 ensures that $f \approx_{K} s_{(\mathbb{N})}$.

Although the hierarchy of antichainable Borel functions under Kakutani reducibility is non-trivial, the following still seems quite plausible:

Conjecture 7.2. Kakutani reducibility of aperiodic, countable-to-one Borel functions is a well-quasi-order.

Perhaps the results of Louveau-Saint-Raymond [13] on Borel linear orders are relevant here. As for Kakutani equivalence, we suggest the following:

Conjecture 7.3. There is no aperiodic Borel function which lies strictly $\leq_{K}$-between $s_{[\mathbb{N}]}$ and $s_{[\mathbb{N}]} \times s_{(\mathbb{N})}$.

A positive solution to this conjecture would completely determine Kakutani equivalence of aperiodic, countable-to-one Borel functions:

Proposition 7.4. Suppose that there is no aperiodic Borel function which lies strictly $\leq_{K}$-between $s_{[\mathbb{N}]}$ and $s_{[\mathbb{N}]} \times s_{(\mathbb{N})}$. Then Kakutani equivalence of aperiodic, countable-toone Borel functions on uncountable Polish spaces is an equivalence relation with exactly seven classes.

Proof. This follows from Proposition 2.2 Lemma 2.13, and Theorem 7.1.

Finally, we mention one more conjecture which is motivated by the sorts of dichotomy theorems that we have proven here:

Conjecture 7.5. Suppose that $X$ is a Polish space and $f: X \rightarrow X$ is an aperiodic, countable-to-one, well-founded Borel function. Then exactly one of the following holds:

(1) The function $f$ is essentially finite-to-one.

(2) There is a Kakutani reduction of $s_{[\mathbb{N}]} \times s_{(\mathbb{N})}$ to $f$.

To see that Conjecture 7.5 implies Conjecture 7.3, observe that if $s_{[\mathbb{N}]}<K \quad f \leq_{K} s_{[\mathbb{N}]} \times$ $s_{(\mathbb{N})}$, then Theorem 5.14 implies that $f$ is not essentially finite-to-one, thus Conjecture 7.5 ensures that $f \simeq{ }_{K} s_{[\mathbb{N}]} \times s_{(\mathbb{N})}$.

Acknowledgments. We began working on the results presented here at the Fields Institute, as visiting graduate students from the University of California, Berkeley, and Université Paris 6, respectively. We would like to express our thanks to all of these institutions for their hospitality and support. We would like to thank also our Ph.D. advisors, Alexander Kechris and John Steel, and Alain Louveau. Finally, we would like to thank Steve Jackson and Charles Boykin for allowing us to include a proof of Theorem 2.6 here, as well as John Clemens, Clinton Conley, and Ilijas Farah for their many useful suggestions.

The first author was supported in part by NSF VIGRE Grant DMS-0502315.

The second author was supported in part by NSF Grant DMS-0556368. 


\section{References}

[1] Boykin, C., Jackson, S.: Some applications of regular markers. In: Logic Colloquium '03, Lecture Notes Logic 24, Assoc. Symbolic Logic, La Jolla, CA, 38-55 (2006) Zbl 1107.03059 MR 2207348

[2] Clemens, J.: Classifying Borel automorphisms. J. Symbolic Logic 72, 1081-1092 (2007) Zbl 1139.03035 MR 2371193

[3] Dougherty, R., Jackson, S., Kechris, A.: The structure of hyperfinite Borel equivalence relations. Trans. Amer. Math. Soc. 341, 193-225 (1994) Zbl 0803.28009 MR 1149121

[4] Farrell, R. H.: Representation of invariant measures. Illinois J. Math. 6, 447-467 (1962) Zbl 0108.14203 MR 0150264

[5] Feldman, J.: Changing orbit equivalences of $\mathbb{R}^{d}$ actions, $d \geq 2$, to be $C^{\infty}$ on orbits. Int. J. Math. 2, 409-427 (1991) Zbl 0760.28014 MR 1130994

[6] Feldman, J.: Correction to: "Changing orbit equivalences of $\mathbb{R}^{d}$ actions, $d \geq 2$, to be $C^{\infty}$ on orbits”. Int. J. Math. 3, 349-350 (1992) Zbl 0773.28013 MR 1163729

[7] Galvin, F., Prikry, K.: Borel sets and Ramsey's theorem. J. Symbolic Logic 38, 193-198 (1973) Zbl 0276.04003 MR 0337630

[8] Harrington, L., Kechris, A., Louveau, A.: A Glimm-Effros dichotomy for Borel equivalence relations. J. Amer. Math. Soc. 3, 903-928 (1990) Zbl 0778.28011 MR 1057041

[9] Jackson, S., Kechris, A., Louveau, A.: Countable Borel equivalence relations. J. Math. Logic 2, 1-80 (2002) Zbl 1008.03031 MR 1900547

[10] Kakutani, S.: Induced measure preserving transformations. Proc. Imp. Acad. Tokyo 19, 635641 (1943) Zbl 0060.27406 MR 0014222

[11] Kechris, A.: Classical Descriptive Set Theory. Grad. Texts in Math. 156, Springer, New York (1995) Zbl 0819.04002 MR 1321597

[12] Kechris, A., Solecki, S., Todorčević, S.: Borel chromatic numbers. Adv. Math. 141, 1-44 (1999) Zbl 0918.05052 MR 1667145

[13] Louveau, A., Saint-Raymond, J.: On the quasi-ordering of Borel linear orders under embeddability. J. Symbolic Logic 55, 537-560 (1990) Zbl 0702.03024 MR 1056369

[14] Miller, D. E.: On the measurability of orbits in Borel actions. Proc. Amer. Math. Soc. 63, 165-170 (1977) Zbl 0349.22001 MR 0440519

[15] Nadkarni, M.: Basic Ergodic Theory. 2nd ed., Birkhäuser, Basel (1998) Zbl 0908.28014 MR 1725389

[16] Ornstein, D., Rudolph, D., Weiss, B.: Equivalence of measure preserving transformations. Mem. Amer. Math. Soc. 37, no. 262 (1982) Zbl 0504.28019 MR 0653094

[17] Rudolph, D.: Smooth orbit equivalence of ergodic $\mathbf{R}^{d}$ actions, $d \geq 2$. Trans. Amer. Math. Soc. 253, 291-302 (1979) Zbl 0446.28019 MR 0536948

[18] Shelah, S., Weiss, B.: Measurable recurrence and quasi-invariant measures. Israel J. Math. 43, 154-160 (1982) Zbl 0554.28020 MR 0689974

[19] Silver, J. H.: Counting the number of equivalence classes of Borel and coanalytic equivalence relations. Ann. Math. Logic 18, 1-28 (1980) Zbl 0517.03018 MR 0568914

[20] Varadarajan, V. S.: Groups of automorphisms of Borel spaces. Trans. Amer. Math. Soc. 109, 191-220 (1963) Zbl 0192.14203 MR 0159923

[21] Wagh, V. M.: A descriptive version of Ambrose's representation theorem for flows. Proc. Indian Acad. Sci. Math. Sci. 98, 101-108 (1988) Zbl 0669.28008 MR 0994127 